\title{
Factores críticos de éxito en aerolíneas de bajo costo en Europa de 1999 a 2018
}

Critical Success Factors (CSF) on low cost airlines in Europe from 1999 to 2018

Fatores críticos de sucesso em companhias aéreas de baixo custo na Europa de 1999 a 2018

Carlos Vázquez Cid de León

Universidad Tecnológica de la Mixteca, México carlosvazquezc@mixtecto.utm.mx https://orcid.org/0000-0003-2067-0565

Ivonne Maya Espinoza

Universidad Tecnológica de la Mixteca, México maya@mixtecto.utm.mx https://orcid.org/0000-0001-9220-4737

Eric Amín Ramírez Castillo

Universidad Autónoma Benito Juárez de Oaxaca, México aminramirez@gmail.com https://orcid.org/0000-0002-8762-1509 


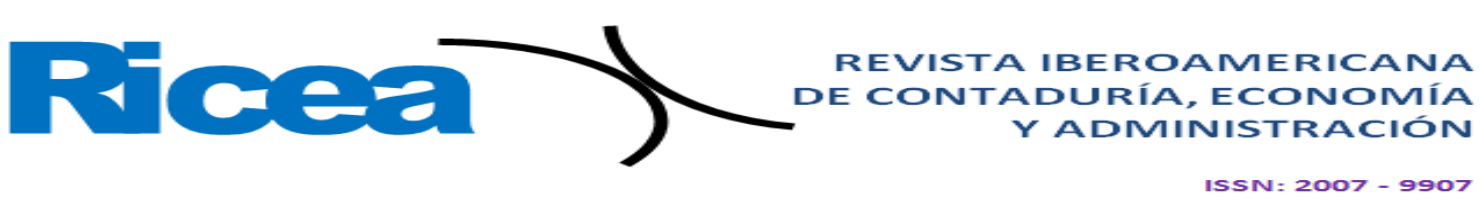

\section{Resumen}

El objetivo principal de este artículo fue analizar los factores críticos de éxito en las aerolíneas de bajo costo europeas. Para ello, primero se realizó un análisis bibliométrico y luego uno estadístico en el cual se aplicaron dos tipos de pruebas (Pearson y técnica R) para verificar si existe una relación entre las variables seleccionadas. En concreto, se trabajó con una muestra de 60 aerolíneas: las más representativas durante el período 1999-2018. Los resultados del estudio muestran la relación entre el aumento en el uso de vuelos de bajo costo y el costo de viaje con características de confort mínimas aceptables.

Palabras clave: aerolíneas, bajo costo, factores críticos de éxito.

\section{Abstract}

The main objective of this article was to analyze a determining factor of success in organizations: the critical success factors in low cost airlines in Europe, in the first part a bibliometric analysis was carried out and in the second statistical one in which They applied two types of tests (Pearson and R technique) to verify if there is a relationship between the selected variables. We worked with a sample of sixty airlines: the most representative during the period from 1999 to 2018 . The results of the study showed the relationship between the increase in the use of low cost flights and the cost of travel with minimum acceptable comfort characteristics.

Keywords: airlines, low cost, critical success factors.

\section{Resumo}

O principal objetivo deste artigo foi analisar os fatores críticos de sucesso nas companhias aéreas europeias de baixo custo. Para isso, foi realizada uma análise bibliométrica e, em seguida, uma estatística, na qual foram aplicados dois tipos de testes (técnica de Pearson e R) para verificar se há relação entre as variáveis selecionadas. Especificamente, trabalhamos com uma amostra de 60 companhias aéreas: as mais representativas durante o período 19992018. Os resultados do estudo mostram a relação entre o aumento no uso de voos de baixo custo e o custo da viagem com características mínimas de conforto aceitáveis.

Palavras-chave: companhias aéreas, baixo custo, fatores críticos de sucesso. 


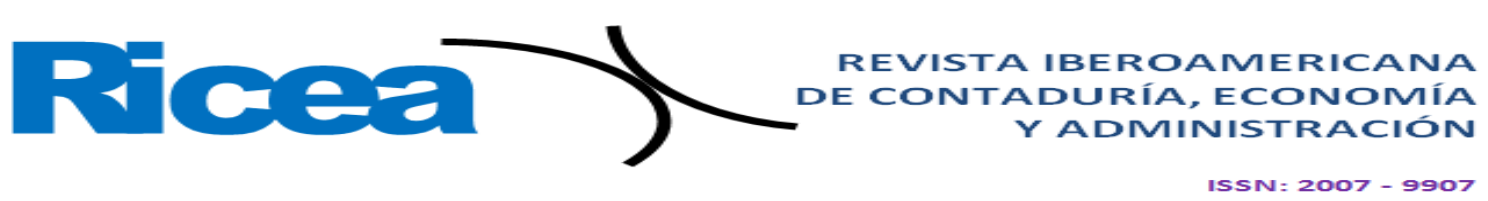

Fecha Recepción: Enero 2019 Fecha Aceptación: Junio 2019

\section{Introducción}

A pesar de las críticas que ha recibido la ventaja competitiva de la teoría Porter (Porter, 1991), la evidencia demuestra que en la actualidad algunas organizaciones siguen implementándola como fórmula estratégica para lograr ocupar un lugar en los mercados internacionales, como sucede con las aerolíneas de bajo costo, conocidas como low cost o low fares airlines.

Por este motivo, el propósito de este trabajo es analizar cómo los factores críticos de éxito de bajo costo (a partir de 1999) han sido usados para generar en las aerolíneas europeas impactos positivos, lo cual fue promovido gracias a la liberalización aérea producida con la llegada de las compañías low cost. Igualmente, se estudian las claves de éxito de esta expansión, así como la evolución de la sensibilidad de los viajeros ante el precio y los impactos que esas compañías han tenido para las compañías aéreas tradicionales. Con esto se intenta determinar si el bajo coste es en realidad una de las claves del éxito en la aceptación de los viajeros.

Al respecto, cabe señalar que a finales de la década de 1970 en Estados Unidos de América se realizó la desregulación económica de las líneas aéreas nacionales con la intención de promover los pasajes económicos (Winston, 1998). No obstante, Southwest Airlines (aerolínea regional) redefinió su estrategia competitiva, lo cual le sirvió no solo para posicionarse como la primera y original aerolínea de bajo coste (low fares carrier o low cost carrier), sino también para convertirse en la línea aérea más rentable de Estados Unidos (European Low Fares Airline Association [ELFAA], 2004).

Este fue un incentivo para que con el paso de los años aparecieran otras aerolíneas de bajo coste en Estados Unidos, lo cual también sucedió a finales de 1980 y durante la década de 1990 en distintos países de Europa (originalmente en Reino Unido e Irlanda), donde las compañías con licencia comunitaria podían atender cualquier ruta internacional dentro de la Unión Europea con una gran libertad en materia de precios (ELFAA, 2004), estrategia que posteriormente se extendió en el sudeste asiático con el movimiento desregulador (Kua y Baum, 2004). 


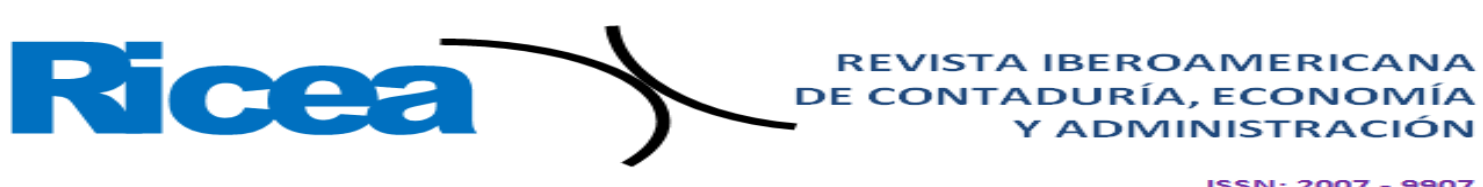

Estos nuevos cambios fueron el sustento para emprender diversas investigaciones, como las desarrolladas por Calder (2002), Ito y Lee (2003), Quirós Tomás (2007, 2008, 2009) y Quirós Tomás y Vega (2011), quienes han profundizado sobre los factores críticos de éxito, entre los que destacan los siguientes: el gran abaratamiento del precio de los boletos desde el surgimiento de las aerolíneas de bajo costo, la correlativa expansión tanto en el número de rutas cubiertas como de vuelos y pasajes disponibles, la atracción de clientes con menor poder adquisitivo, el análisis de los niveles de renta de viajeros internacionales a España en compañías tradicionales y de bajo coste (Subdirección General de Conocimiento y Estudios Turísticos, 2013).

Sobre este creciente fenómeno comercial, se estima que en 2005 tan solo en Europa existían 60 compañías de vuelos de esta índole conocidas como compañías de bajo costo (CBC), las cuales empelan estrategias competitivas completamente distintas a las usadas por las compañías tradicionales (Quirós y Vega, 2011), las cuales se basan en una propuesta de Porter (1980): el liderazgo en costos.

Esas 60 líneas se encontraban en 15 países de toda Europa y representaban $25 \%$ del total de ocupación, con un promedio mundial de crecimiento de 4.6\% (Banco Mundial [BM], 2013). Las compañías de bajo costo alrededor del mundo han jugado un papel importante en la extraordinaria expansión del sector en el último cuarto de siglo. Para 2013, estas transportaron $31 \%$ del total de pasajeros en el mundo (International Civil Aviation Organization [ICAO], 2014).

De la Fuente y Muñoz (2003) plantean que el origen de la ventaja competitiva radica en las actividades de la cadena de valor —enfoque ampliamente defendido por Porter (1991)—, así como en los recursos — idea formulada por Wernerfelt $(1984,1995)$ — De la Fuente y Muñoz (2003) analizan estos dos enfoques y con base en ello formulan una propuesta general que consideran debe ser validada en otras investigaciones.

Al respecto, no obstante, vale comentar que las $\mathrm{CBC}$ tienen una relación con los estudios reportados sobre ventaja competitiva y que el artículo más citado es el de Porter (2007) en cuanto a la ventaja competitiva basada en el costo, estrategia que sigue siendo aprovechada por muchas organizaciones en 2018, razón por la que aún se debe seguir investigando sobre este tema. 


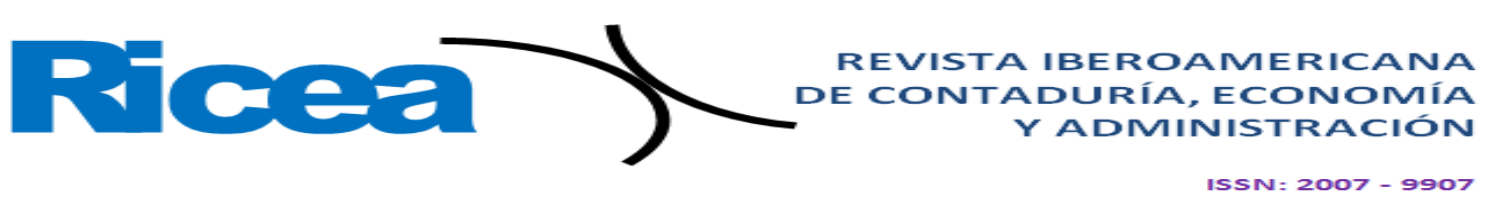

La estrategia competitiva, desde la perspectiva de Porter (2000), es la acción que lleva a desarrollar una amplia fórmula de cómo la empresa va a competir y cuáles deberían ser sus objetivos y políticas para alcanzar dichos objetivos. Porter afirma que la estrategia competitiva es una combinación de los fines (metas) por los cuales se está esforzando la empresa y los medios (políticas) con las cuales se está buscando llegar a ellos. Tomando como base este enfoque, Porter propone un axioma que sugiere que las empresas pueden llevar a cabo tres estrategias competitivas genéricas: diferenciación, enfoque y liderazgo en costos (sobre esta última se enfoca la presente investigación).

La estrategia de liderazgo en costos se justifica desde cuatro condiciones: cuando los consumidores de determinadas industrias son sensibles a los precios, cuando existen pocos cambios para lograr diferencias entre los productos, cuando a los compradores no les interesa las diferencias entre una marca y otra, o cuando existe una gran cantidad de compradores con un considerable poder de negociación.

Sin embargo, según Lizzi (2004), la ventaja competitiva de Porter se sustenta en tener la primacía en la posición del mercado de operación, para lo cual el costo indiscutiblemente es un factor de éxito. Aun así, Porter (1980) menciona que el liderazgo por costos se sustenta en cuatro puntos: 1) lograr el liderazgo por costo significa que una firma se establece como el productor de más bajo costo en su industria; 2) un líder de costos debe lograr paridad, o por lo menos proximidad, aun cuando confíe en el liderazgo de costos para consolidar su ventaja competitiva; 3) si más de una compañía intenta alcanzar el liderazgo por costos al mismo tiempo, este es generalmente desastroso, y 4) el uso de economías a escala.

En este contexto, por supuesto, también puede suceder que las aerolíneas low cost que utilicen estrategias hibridas combinen el bajo costo y elementos de diferenciación (Kim, Nam y Stimpert, 2004). Para Hernández (2000, citado por Quero, 2008) la competitividad se relaciona con una mejora continua que se debe desarrollar a lo largo de una trayectoria que cada empresa debe transitar, aunque de forma resumida se puede decir que su objetivo se centra en tener los menores costos posibles mientras se enfocan en determinadas rutas, las cuales de forma habitual se encuentran en constante crecimiento. Un ejemplo de lo explicado son los costes por asiento y kilómetro ofertado (AKO) que había en 2005 para rutas intraeuropeas, pues mientras que en las compañías de bajo coste había una diferencia de entre 4 y 7 céntimos de euro, en las compañías tradicionales esa diferencia se encontraba entre 9 y 12 céntimos de euro (European Cockpit Association [ECA], 2006). 


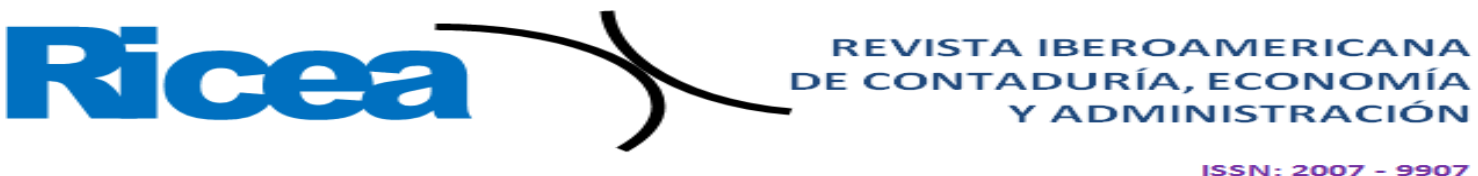

Finalmente, también hay casos en los que se ha estudiado la ganancia según el número de asientos que ofrecen las aerolíneas tradicionales y las de bajo costo. Los resultados, sin embargo, reflejan números muy poco alentadores, por lo que se requiere de una mejora continua en los gastos de operación para poder asegurar el éxito de las compañías. La tabla 1 presenta una distribución de McCartney (15 de junio de 2012) publicada en Wall Street Journal en torno a la distribución de asientos y los costos que cubren a partir de un vuelo hipotético de 100 pasajeros.

Tabla 1. Distribución de asientos y costo que cubren

\begin{tabular}{|l|l|}
\hline $\begin{array}{l}\text { Número de } \\
\text { asientos }\end{array}$ & Costo que cubre el asiento \\
\hline 29 & Cubren el costo del combustible \\
\hline 20 & Sueldos \\
\hline 16 & Al avión \\
\hline 14 & Impuestos \\
\hline 11 & Mantenimiento \\
\hline 9 & Se perdían en la cuenta de otros \\
\hline 1 & Ganancia del vuelo \\
\hline
\end{tabular}

Fuente: Elaboración propia con datos de McCartney (2012).

En 2013, la red mundial de aerolíneas transportó 3.1 billones de pasajeros en 33 millones de vuelos programados, cifras que según diversos estudios se podrían duplicar para el año 2030, de ahí que dicha actividad cumpla un papel muy importante en la economía mundial (Airbus, 2014; Eurocontrol, 2014; ICAO, 2014).

\section{Método}

La presente investigación fue de tipo cuantitativa, exploratoria, descriptiva y correlacional, lo cual sirvió para realizar un aporte en torno a cómo se distribuye la producción científica del tema de estudio. Para el análisis de la información se utilizó la bibliometría, técnica empleada para el análisis cuantitativo de las publicaciones científicas (López, citado por Camps y Chauhan, 2009). En relación con los indicadores bibliométricos, los parámetros fueron categorías de búsqueda en torno a la productividad de los autores o instituciones, la cual se puede medir según los siguientes criterios: número de trabajos publicados, crecimiento de cualquier campo de la ciencia, variación cronológica del número de trabajos divulgados y colaboración entre los científicos o instituciones, así como número 


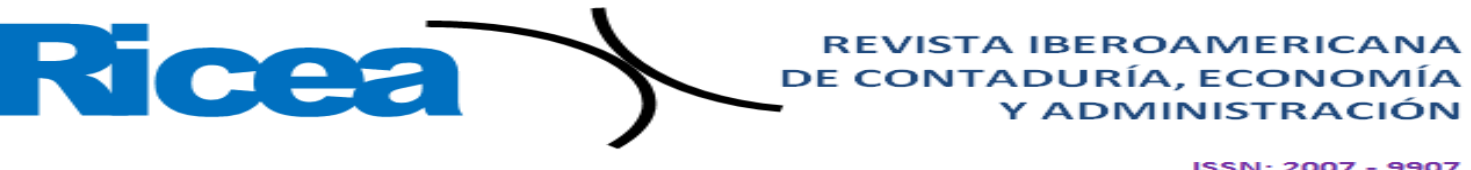

de autores por trabajo o centros de investigación que colaboran (Sancho, 1990). En concreto, se plantearon los siguientes objetivos específicos de investigación:

- Realizar una revisión bibliométrica para dar soporte a un trabajo de investigación transeccional descriptivo y exploratorio sobre las aerolíneas low cost europeas durante el periodo 1999-2018.

- Seleccionar uno de los factores críticos de éxito de las organizaciones para caracterizarlo en un trabajo de investigación documental transeccional.

- Desarrollar un análisis estadístico que permita verificar el cumplimiento de alguna de las hipótesis planteadas para aportar nueva información a la comunidad interesada.

Ahora bien, como ya se ha mencionado, el éxito de las aerolíneas de bajo costo se sustenta, entre otros factores, en que su modelo de negocios se basa en la reducción de las tarifas para el pasajero (Binggeli y Pompeo, 2002). Estos costos se pueden dividir en tres categorías (Belobaba, Odoni y Barnhart, 2009):

1. Costos de operación de vuelo (por sus siglas en ingles FOC3): Estos comprenden cerca de $54 \%$ del total de costos que incluyen las operaciones de vuelo (los costos de operación del sistema representan $26 \%$, mientras que los costos de operación en tierra suman aproximadamente $20 \%$ del total de los gastos; el $8 \%$ restante se debe a otros costos).

2. Mantenimiento.

3. Depreciación y amortización ocasionadas principalmente por las fluctuaciones en los precios del combustible.

Igualmente, se debe recordar que las aerolíneas clásicas usan diversos tipos de aviones según los vuelos y destinos, mientras que las low cost solo emplean un tipo de aeronave. Esto reduce los costos porque unifica los criterios de capacitación de las asistentes de vuelo o azafatas, así como de los pilotos y del personal de mantenimiento técnico y mecánico. Además, se utilizan modelos de aviones que no tienen asientos reclinables para reducir en la compra de refacciones, así como en los ajustes para mantenerlos en óptimas condiciones. De hecho, no tienen un compartimento de portaobjetos debajo del asiento (lo que facilita la limpieza del avión y, en consecuencia, disminuye los tiempos de espera y las penalizaciones por viajes retrasados) y tampoco cuentan con sistemas de entretenimiento. Todo ello contribuye a que las aeronaves de las compañías low cost permanezcan más tiempo en el aire con un número menor de empleados (30 o 40 personas, a diferencia de los más de 


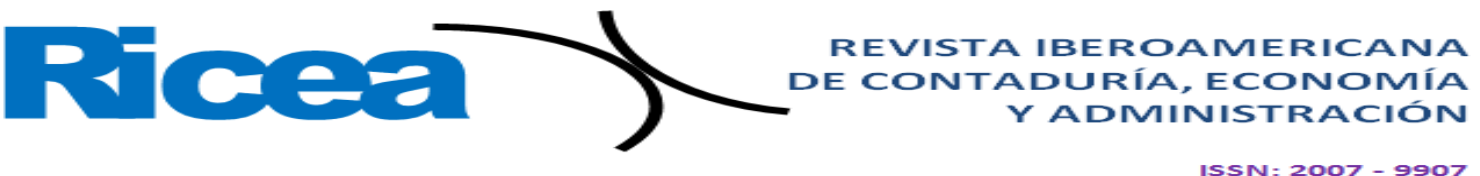

100 empleados de las aerolíneas tradicionales), lo que hace más competitiva a la empresa y le permite conseguir más ganancias.

Para ilustrar mejor esta idea, a continuación se presenta una estructura de costos de aerolíneas mexicanas (figura 1), la cual se puede comparar con las europeas (figura 2):

Figura 1. Estructura de costes en aerolíneas tradicionales mexicanas

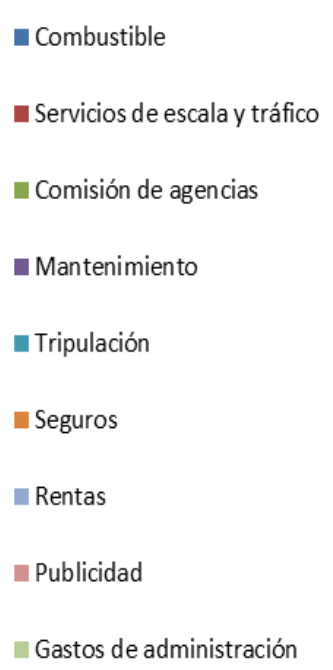

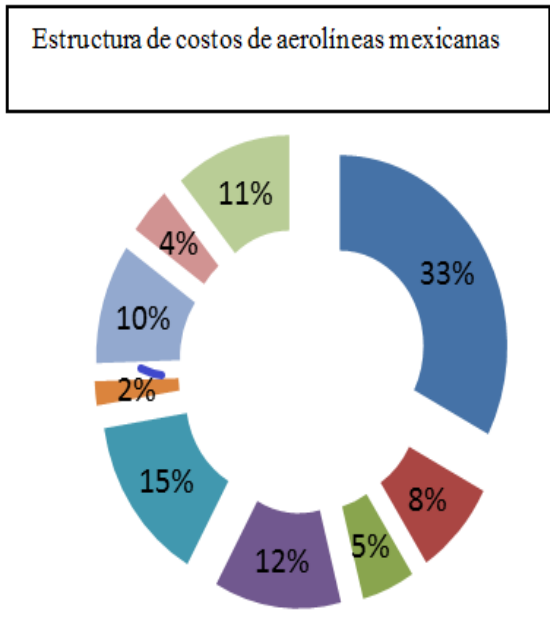

Fuente: Elaboración propia con base en los datos de Canseco, Zúñiga y Blanco (2015) y la Secretaría de Comunicación y Transporte (SCT) (2013).

Figura 2. Estructura de costes en aerolíneas de tipo tradicional en Europa
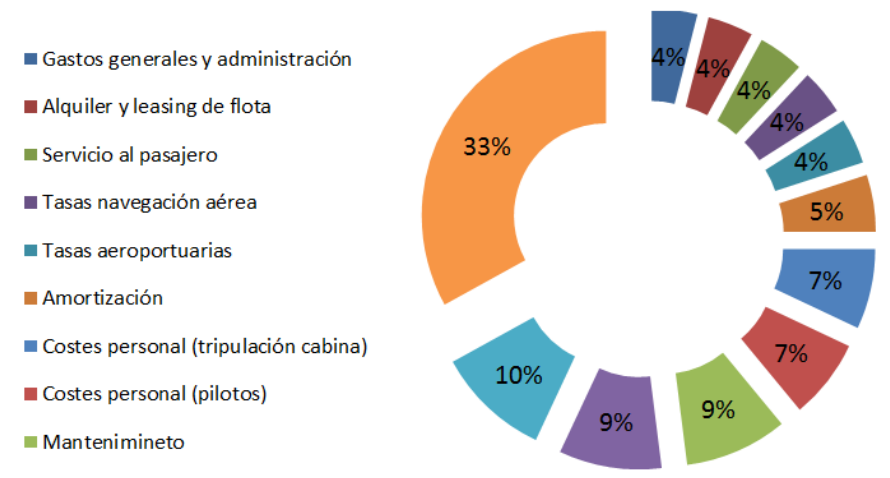

Fuente: Secretaría de Estado de Transportes (2008).

Como se puede observar, aunque en ambos casos el combustible representa $33 \%$ del costo, los indicadores de operación cambian en cuanto a ahorros, pues las CBC tienen más ventaja que las compañías de bandera (gubernamentales o privatizadas). 
Otro ejemplo se presenta en la figura 3, donde se evidencia la estructura de costes de Ryanair frente a una compañía de bandera en Europa:

Figura 3. Estructura de costes de Ryanair frente a una compañía de bandera en Europa

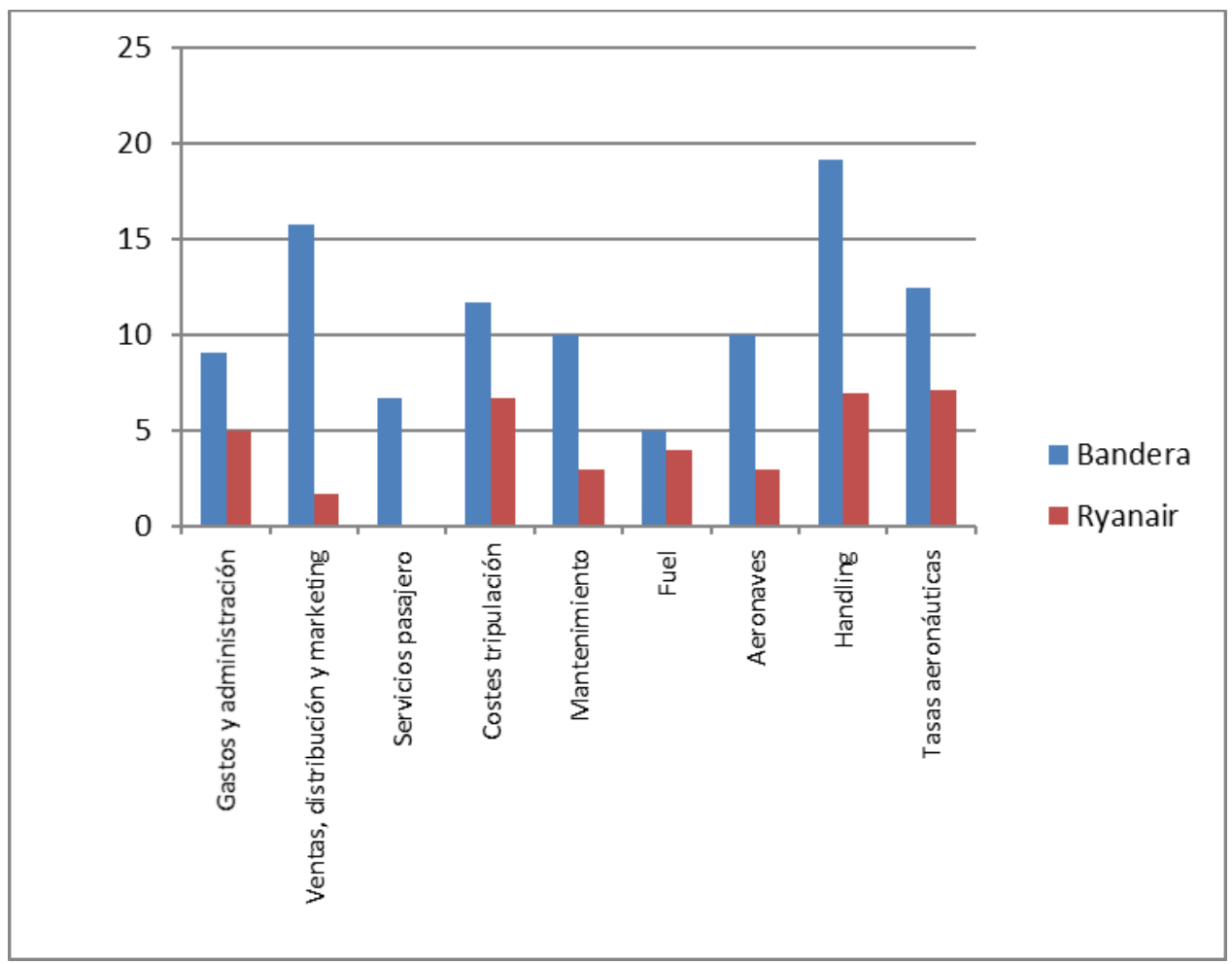

Fuente: Elaboración propia con datos de Candela-Garriga (2008).

Por otra parte, en la figura 4 se muestra la reducción de costes de las compañías europeas low cost (valores en \%), lo que ofrece un panorama más claro sobre los avances en esa materia, entre los que se destaca la menor densidad en butacas (16\%). 


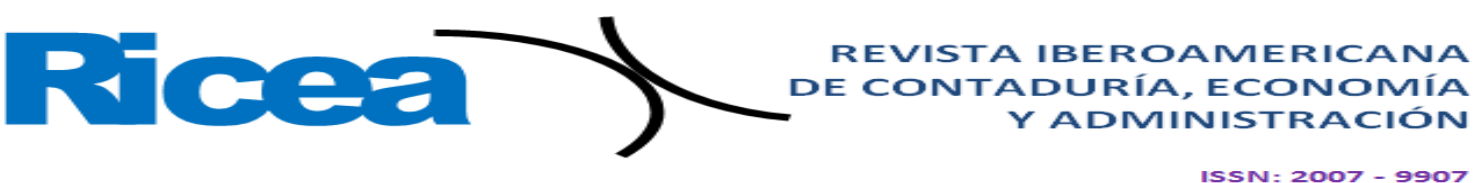

Figura 4. Reducción de costes de las compañías low cost en Europa (valores en \%)

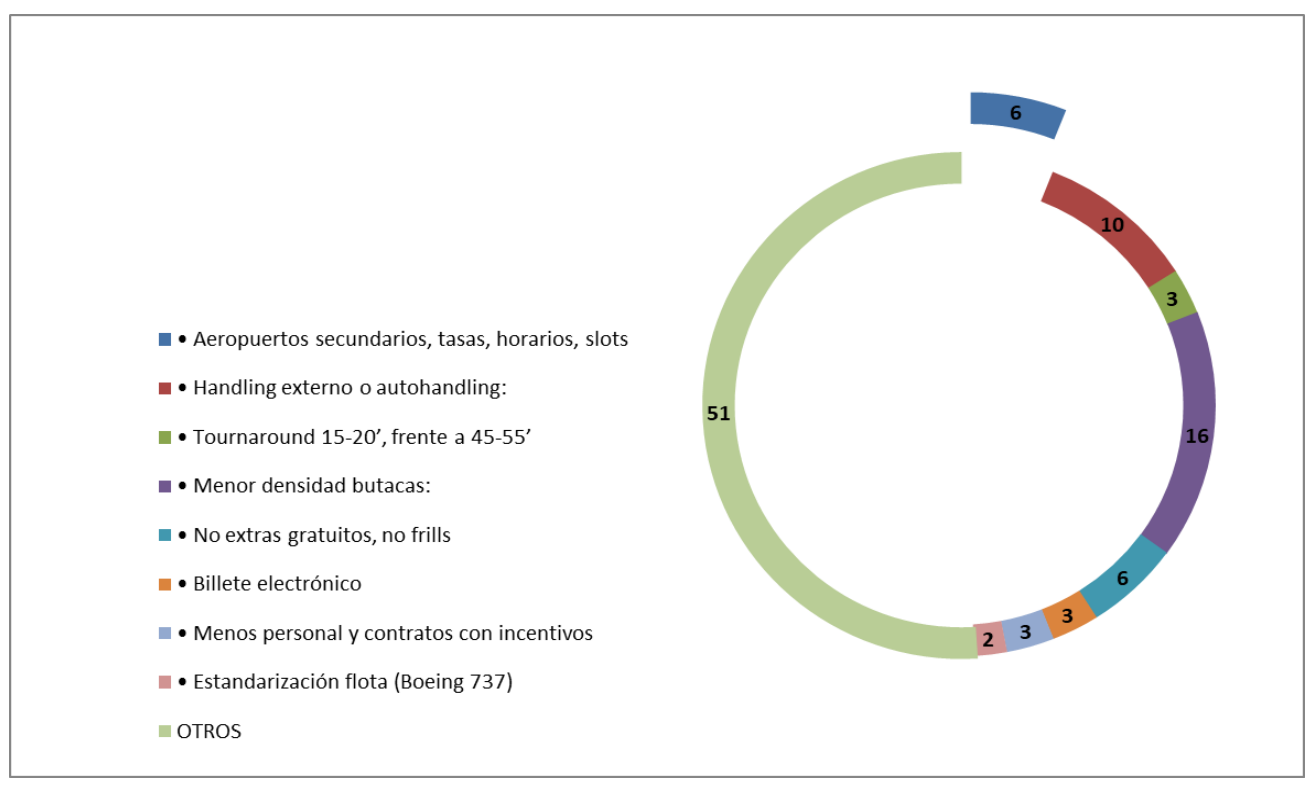

Fuente: Elaboración propia con datos de la The European Cockpit Association (Francesc, 2008).

El fenómeno del bajo coste ha producido un efecto fundamental: ha atraído a nuevos viajeros, los cuales hasta el momento no podían acceder a ese medio de transporte. En efecto, actualmente la demanda pasajeros se calcula en $59 \%$, de los cuales $71 \%$ no viajaba anteriormente porque lo hacían, por ejemplo, en coche (15\%), en tren (6\%) o en otros medios (8 \%) (Skeels, 2005a, 2005b, 2005c).

Ahora bien, para señalar la hipótesis planteada, se presenta el ritual de la significancia estadística usado (tabla 2): 


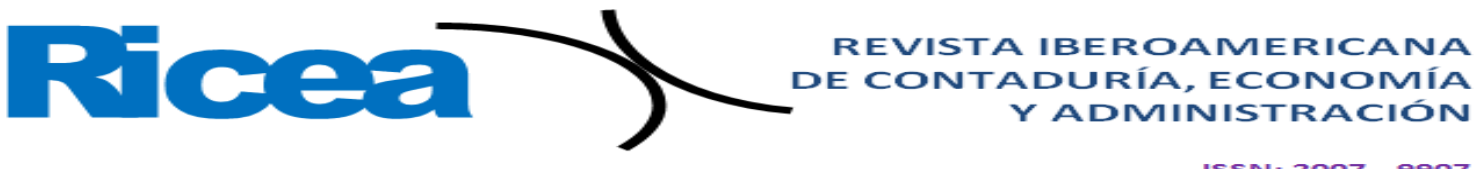

Tabla 2. Ritual de la significancia estadística

\begin{tabular}{|l|l|}
\hline 1 & $\begin{array}{l}\text { Plantear hipótesis. Ho: No existe correlación entre el éxito y el costo por vuelo } \\
\text { H1: Existe correlación entre el éxito y el costo por vuelo }\end{array}$ \\
\hline 2 & Establecer un nivel de significancia. Nivel de significancia alfa $\alpha=5 \%=0.05$ \\
\hline 3 & $\begin{array}{l}\text { Selección del estadístico de prueba } \\
\text { Correlación de Pearson } \\
\text { Correlación de Spearman (aplicable a diferencia de rangos) } \\
\text { Técnica R } \\
\text { Análisis de la Varianza Anova (se descarta por ser aplicable a una variable } \\
\text { dependiente y por ser usadas en muestras que tienen la misma media) } \\
\text { Alfa de crombach (no se considera porque no se utilizó un instrumento para la } \\
\text { recolección ni para validarlo) }\end{array}$ \\
\hline 4 & $\begin{array}{l}\text { Sea } \alpha=0.05 . \text { Spearman } r=0.9449 \text { rango de relación }+0.91 \text { a }+1.00 \text { Correlación } \\
\text { positiva perfecta } \\
\text { Pearson = 0.9449R =0.8929 }\end{array}$ \\
\hline 5 & $\begin{array}{l}\text { Toma de decisión: Es un determinante H1: Existe correlación entre el éxito y el } \\
\text { costo por vuelo }\end{array}$ \\
\hline
\end{tabular}

Fuente: Elaboración propia.

En cuanto a los datos y las operaciones, cabe destacar que el término estadística no paramétrica hace referencia a los métodos estadísticos que no requieren la especificación de un supuesto sobre la distribución de la que proceden los datos de la muestra para efectuar inferencias sobre la población (Cáceres, 2006). Es decir, no exigen la suposición de la normalidad de la población de la cual fue extraída la muestra.

Asimismo, para el análisis de datos en forma estadística no paramétrica se tienen los siguientes supuestos: la muestra disponible para el análisis de las variables $X$ y $Y$ son medidas en, por lo menos, una escala ordinal (Anderson, Sweeney y Williams, 1999); así, se tiene un $\alpha=0.05, \mathrm{n}=6$ pares de datos de las compañías de bajo costo en la primera prueba y en la segunda prueba $\mathrm{n}=11$ pares de datos de las compañías tradicionales, $\mathrm{X}=$ años estudiados $\mathrm{y}$ $\mathrm{Y}=$ incremento en los vuelos, para poder comprobar su correlación.

La primera prueba de Spearman (tabla 3) se realizó de manera análoga y la prueba de Pearson en software Excel; en el primer caso se comprobó que existe correlación positiva perfecta, mientras que en la segunda prueba (realizada en Excel) se determinó su correlación positiva media, como se muestra a continuación:

Fórmula de Spearman:

En donde:

$\mathrm{r}=$ coeficiente de correlación

$\mathrm{n}=$ número de pares ordenados 
$\mathrm{x}=$ variable independiente

$\mathrm{y}=$ variable independiente

Tabla 3. Datos para la prueba de Spearman

\begin{tabular}{|c|c|c|c|c|}
\hline año (x) & Incremento $(y)$ & $\mathrm{X}^{2}$ & $\mathrm{Y}^{2}$ & $\mathrm{X}^{*} \mathrm{Y}$ \\
\hline 1999 & 13000 & 3996001 & 169000000 & 25987000 \\
\hline 2000 & 17000 & 4000000 & 289000000 & 34000000 \\
\hline 2001 & 24000 & 4004001 & 576000000 & 48024000 \\
\hline 2002 & 39000 & 4008004 & 1521000000 & 78078000 \\
\hline 2003 & 47000 & 4012009 & 2209000000 & 94141000 \\
\hline 2004 & 80000 & 4016016 & 6400000000 & 160320000 \\
\hline 12009 & 220000 & 24036031 & 11164000000 & 440550000 \\
\hline
\end{tabular}

Fuente: Elaboración propia.

$r=\frac{n \sum_{i=1}^{n} x_{i} y_{i-} \sum_{i=1}^{n} x_{i} * \sum_{i=1}^{n} y_{i}=}{\left.\sqrt{\left(n \sum_{i=1}^{n} x_{i}^{2}-\left(\sum_{i=1}^{n} x_{i}\right)\right.} 2\right)\left(n \sum_{i=1}^{n} y_{i}^{2}-\left(\sum_{i=1}^{n} y_{i)} 2\right)\right.} \frac{6 * 440550000-(1209 * 220000)=}{\sqrt{(6 * 24036031-(12009) 2)(6 * 11164000000-(220000) 2)}} 0.944951858$

$\mathrm{r}=0.9449$ rango de relación $+0.91 \mathrm{a}+1.00$ Correlación positiva perfecta

La interpretación de los valores ha sido expresada por diversos autores en escalas, una de las cuales (la más usada) se presenta en la tabla 4: 


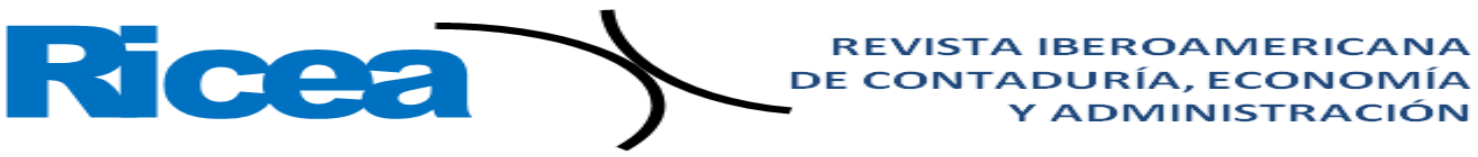

ISSN $=2007-9907$

Tabla 4. Grado de relación según coeficiente de correlación

\begin{tabular}{|l|}
\hline Rango relación \\
\hline-0.91 a -1.00 Correlación negativa perfecta \\
\hline-0.76 a -0.90 Correlación negativa muy \\
fuerte \\
\hline-0.51 a $\quad-0.75$ Correlación negativa \\
considerable \\
\hline-0.11 a -0.50 Correlación negativa media \\
\hline-0.01 a -0.10 Correlación negativa débil \\
\hline 0.00 No existe correlación \\
\hline+0.01 a +0.10 Correlación positiva débil \\
\hline+0.11 a +0.50 Correlación positiva media \\
\hline$+0.51 \quad$ a $+0.75 \quad$ Correlación positiva \\
considerable \\
\hline $\begin{array}{l}+0.76 \text { a }+0.90 \text { Correlación positiva muy } \\
\text { fuerte }\end{array}$ \\
\hline+0.91 a +1.00 Correlación positiva perfecta \\
\hline
\end{tabular}

Fuente: Elaboración propia con datos de Hernández, Fernández y Baptista (1998)

A continuación, se presentan las figuras 5 y 6 de los análisis de Pearson y R con los datos disponibles de las aerolíneas de bajo costo y tradicionales durante dos periodos (en la figura 5 aparecen las empresas de bajo costo de 1999 a 2004 y en figura 6 las mismas compañías pero en el periodo de 2005 a 2015, así como los resultados de su prueba estadística). 


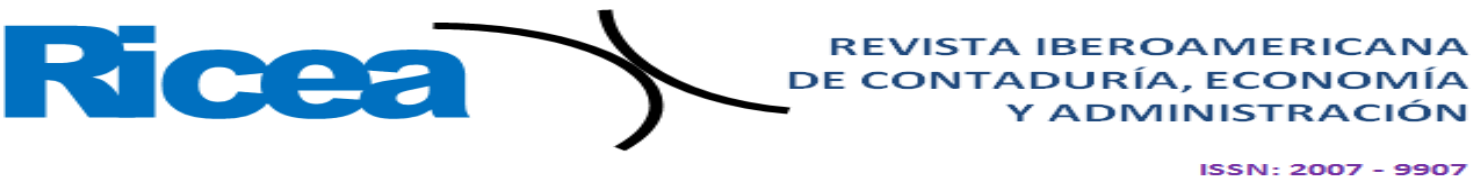

Figura 5. Prueba de Pearson realizada a compañías de bajo costo (1999-2004)

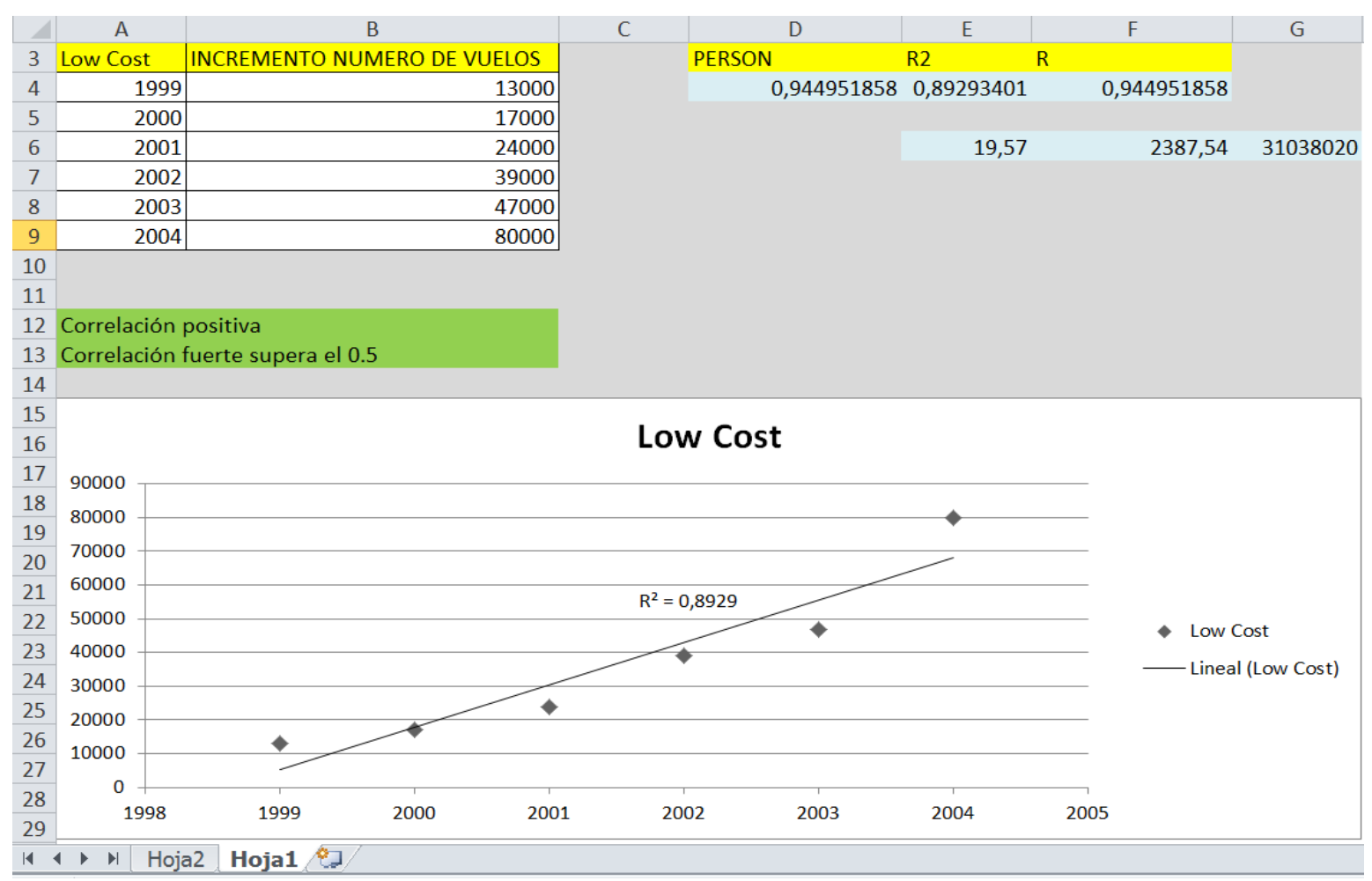

Fuente: Elaboración propia con datos de ELFAA (2005)

Como se puede apreciar, la correlación es positiva y con un resultado perfecto en el caso de las compañías de bajo costo, mientras que en los resultados de las compañías tradicionales la correlación positiva es media. Esto significa que el éxito de las compañías se puede hallar en otras variables o factores que tienen una correlación más fuerte, por lo que no fue necesario hacer la prueba de Spearman de manera análoga. 


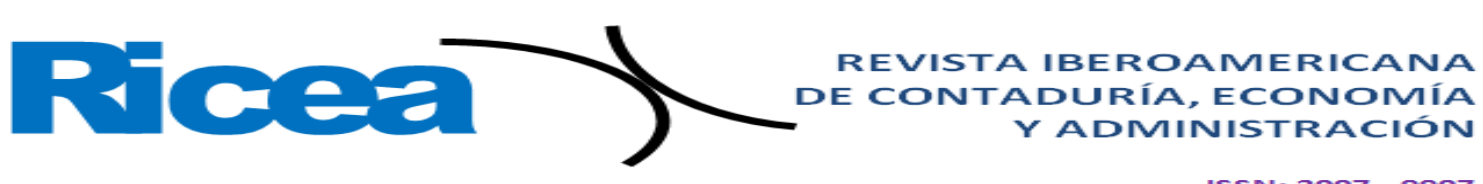

Figura 6. Prueba de Pearson realizada a compañías de bajo costo (2005-2015)

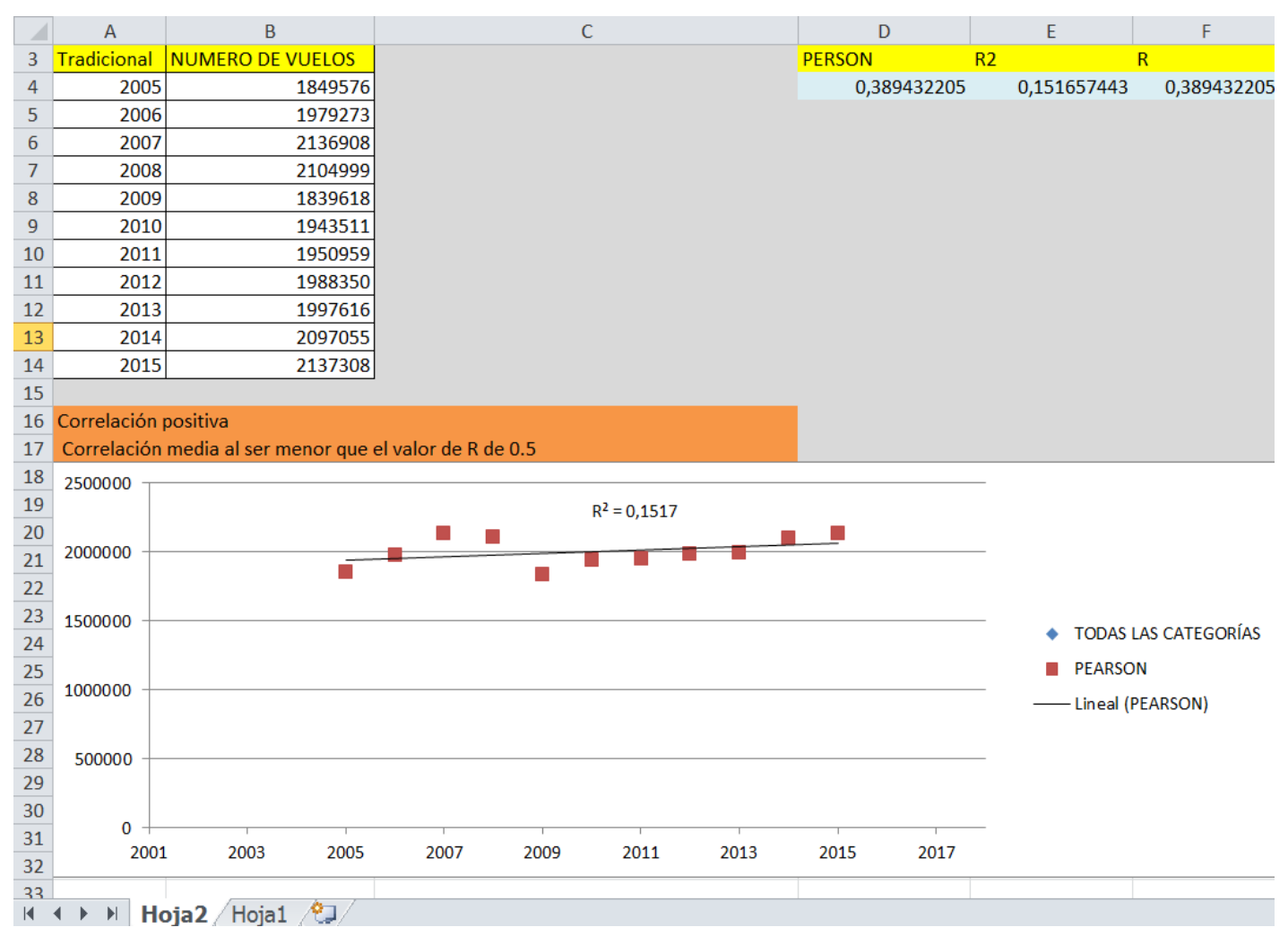

Fuente: Elaboración propia con datos de ELFAA (2016).

Se puede concluir que la toma de decisión es un determinante H1, es decir, existe correlación entre el éxito y el costo por vuelo, aunque para comprobar el caso de las compañías tradicionales se requiere de otro estudio.

\section{Discusión y resultados}

De acuerdo con Delgado López-Cózar, Ruiz y Jiménez (2006), las revistas científicas son un canal que les permite a los profesionales e investigadores de diferentes disciplinas dar a conocer sus hallazgos, intercambiar experiencias o informarse sobre nuevos avances y descubrimientos.

En este sentido, vale recordar que uno de los objetivos de este estudio fue hacer una revisión en las bases de datos de Scopus sobre la producción científica publicada de manera genérica en torno a Critical success factors frente a lo publicado sobre Critical success factors on low cost airlines in Europe from 1999 to 2018, aunque para este último criterio se usó la expresión Critical success factors on low cost airlines para evitar que los resultados pudieran nulos. 


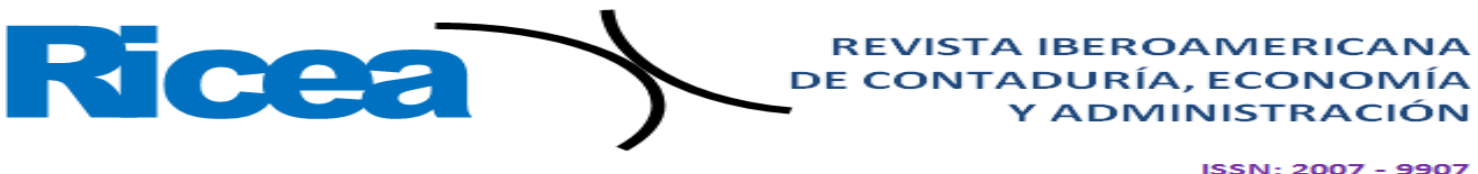

Asimismo, para la recolección de la información se elaboró el análisis de indicadores bibliométricos, los cuales fueron clasificados en siete categorías según los documentos 1) generados por año, 2) por año y por fuente, 3) por autor, 4) por filiación institucional, 5) por país, 6) por tipo de documento y 7) por área del conocimiento.

La Figura 7 pertenece a los resultados de Critical success factors, mientras que la Figura 8 corresponde a los resultados de la búsqueda Critical success factors on low cost airlines. Al respecto, vale acotar que en algunos casos aparecen áreas de conocimiento distintas a las del tema central de este trabajo, pero igualmente se tomaron en cuenta para contar con un parámetro de comparación.

\section{Categoría 1: Documentos generados por año}

Los resultados para la búsqueda Critical success factors fueron 15931 documentos (en el año 2017 hubo la mayor producción con 1309 documentos), mientras que para Critical success factors on low cost airlines solo se hallaron 2 documentos: uno publicado en 2004 — titulado Aeropuertos regionales: estudio de caso de sostenibilidad, supervivencia o éxito por la International Engineering Management Conference (IEEE) - y otro divulgado en 2018 - cuya fuente es la Quality Engineering, dedicado a la mejora del flujo de equipaje con las herramientas Lean Six Sigma en una aerolínea estadounidense - .

Figura 7. Resultados de la búsqueda Critical success factors

Documents by year

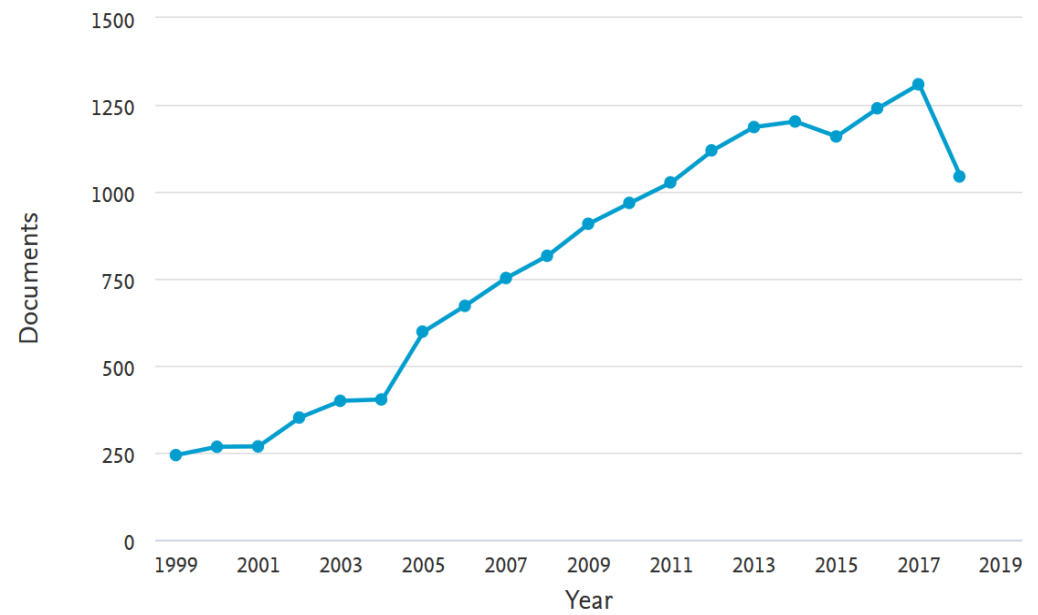

Fuente: Elaboración propia con datos de Scopus (2018). 
Figura 8. Resultados de la búsqueda Critical success factors on low cost airlines

Documents by year

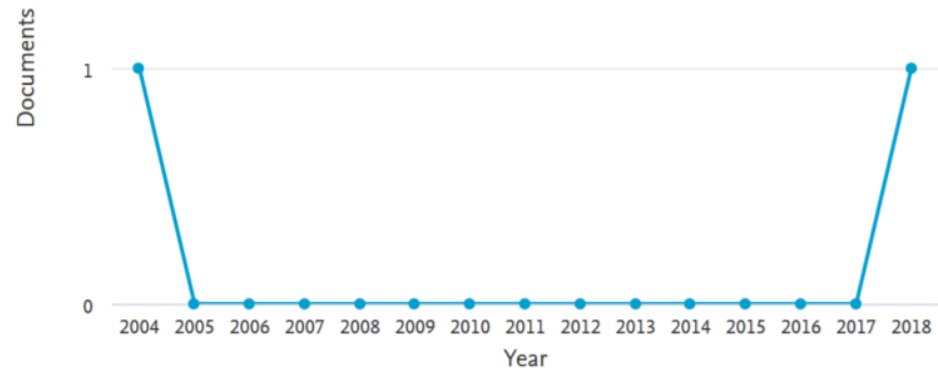

Fuente: Elaboración propia con datos de Scopus (2018).

\section{Categoría 2: Documentos generados por año y por fuente}

En la figura 9 se aprecia que en 2012 hubo la mayor producción con 12 documentos, mientras que en la figura 10, se observan otros documentos generados por fuente, siendo estos dos artículos con Scimago Journal Ranking (SJR) de 0.104 y 0.804, respectivamente. Este es un indicador que muestra la visibilidad de las revistas contenidas en Scopus, así como un Source Normalized Impact per Paper (SNIP), que mide el impacto de una cita. En tal sentido, las características de la materia investigada tuvieron valores de 0.007 y 0.969 , respectivamente, lo cual resulta muy interesante, aunque también demuestra que son pocos los resultados en cuanto a la temática de interés principal. 
Figura 9. Documentos generados, por año y fuente, en torno al criterio Critical success

\section{factors}

Documents per year by source

Compare the document counts for up to 10 sources

Compare sources and view CiteScore, SJR, and SNIP data

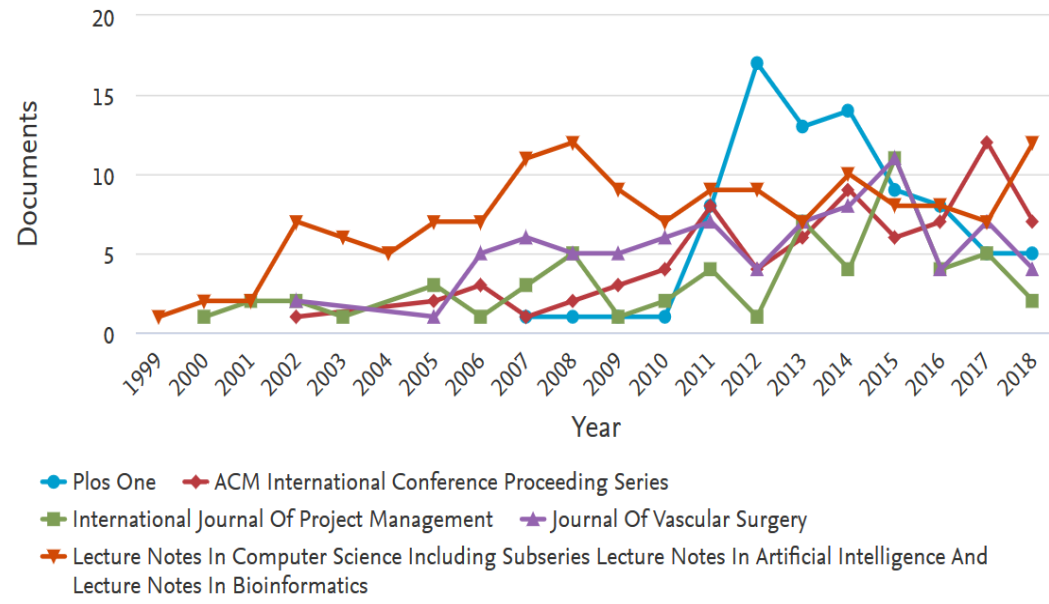

Fuente: Elaboración propia con datos de Scopus (2018).

Figura 10. Otros documentos generados por fuente, en torno al criterio Critical success factors

Documents per year by source Compare the document counts kse up to 10 sounces

Compare sources and vire Cite5eore, SjR, and SNIP data

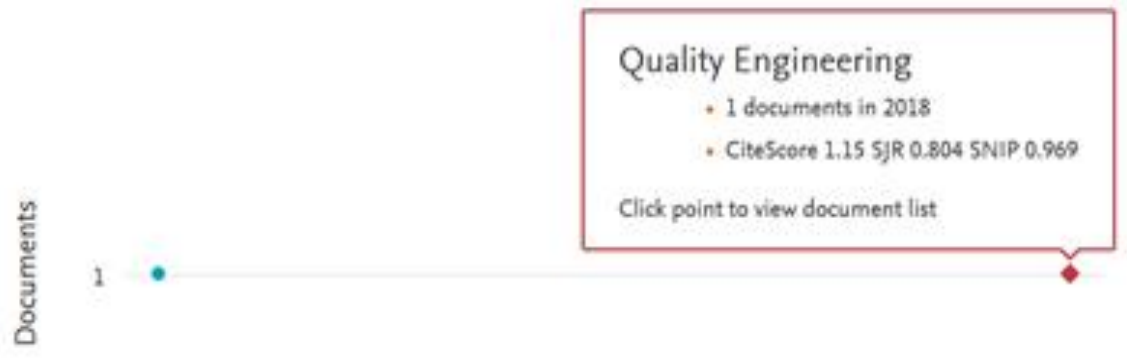

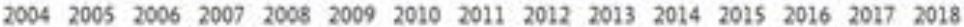

Year

- IEEE International Engineering Management Coeference +Quality Engineering

Fuente: Elaboración propia con datos de Scopus (2018). 


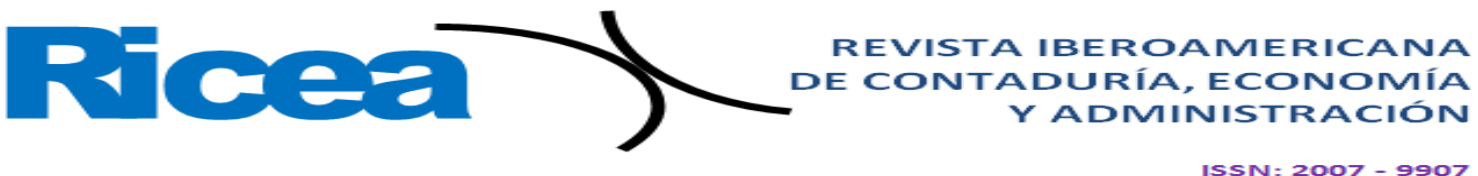

\section{Categoría 3: Documentos generados por autor}

En la figura 11 se observa que Chan tiene 39 documentos, de los cuales Factors affecting the success of a construction project —en la fuente Journal of Construction Engineering and Managementel - es el más reseñado con 284 citas.

Igualmente, Haleem cuenta con 13 documentos, de los cuales el más referenciado con 84 citas es Analysis of critical success factors of world-class manufacturing practices: An application of interpretative structural modelling and interpretative ranking process, cuya fuente es Production Planning and Control.

Por otra parte, en la figura 12 se aprecian los autores que tienen un solo documento. En el caso de Al-Ashi, su artículo Improving baggage flow in the baggage handling system at a UAE-based airline using lean Six Sigma tools no había sido referenciado hasta el 15 de noviembre de 2018.

Figura 11. Documentos por autores más citados para el criterio Critical success factors

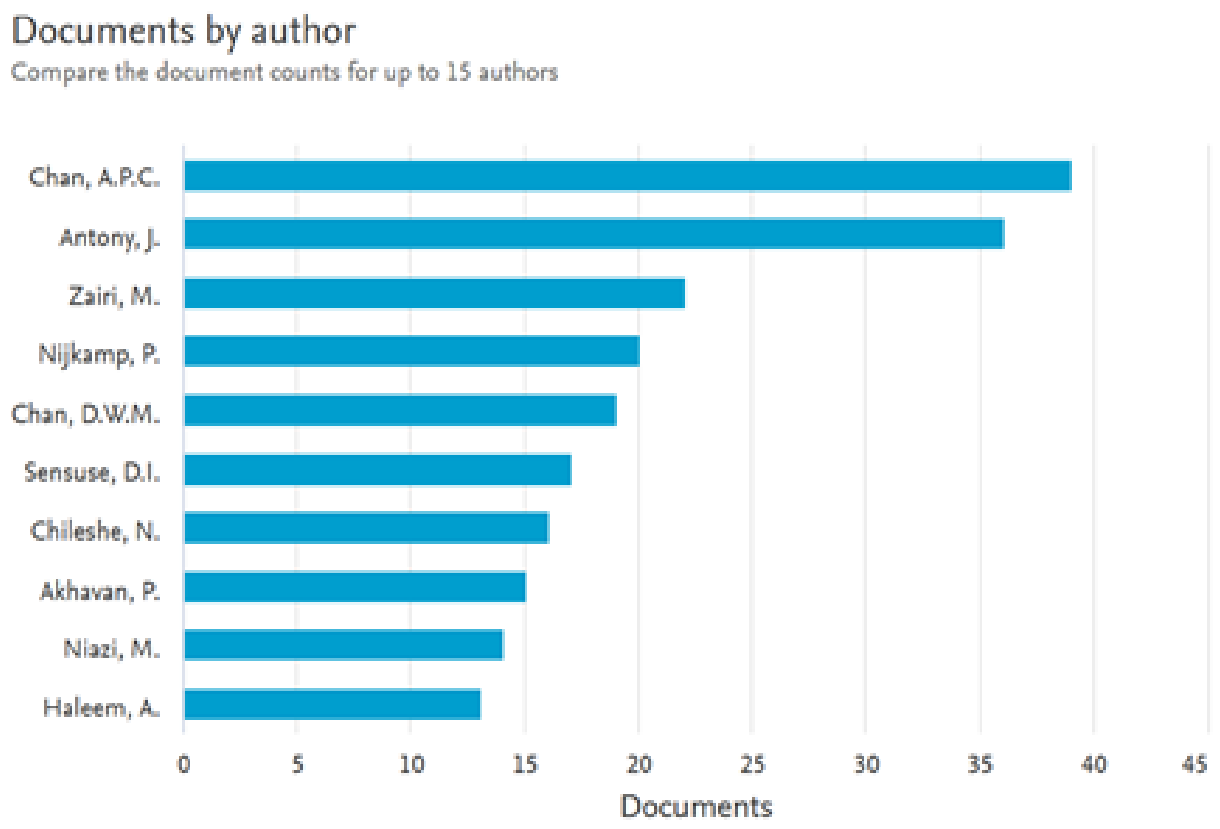

Fuente: Elaboración propia con datos de Scopus (2018). 


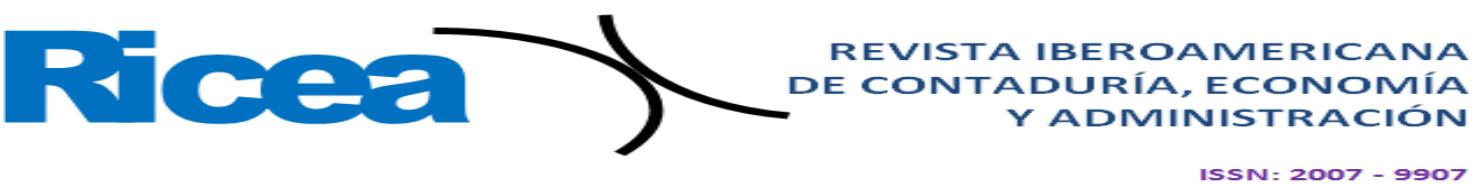

Figura 12. Documentos por autores menos citados para el criterio Critical success factors

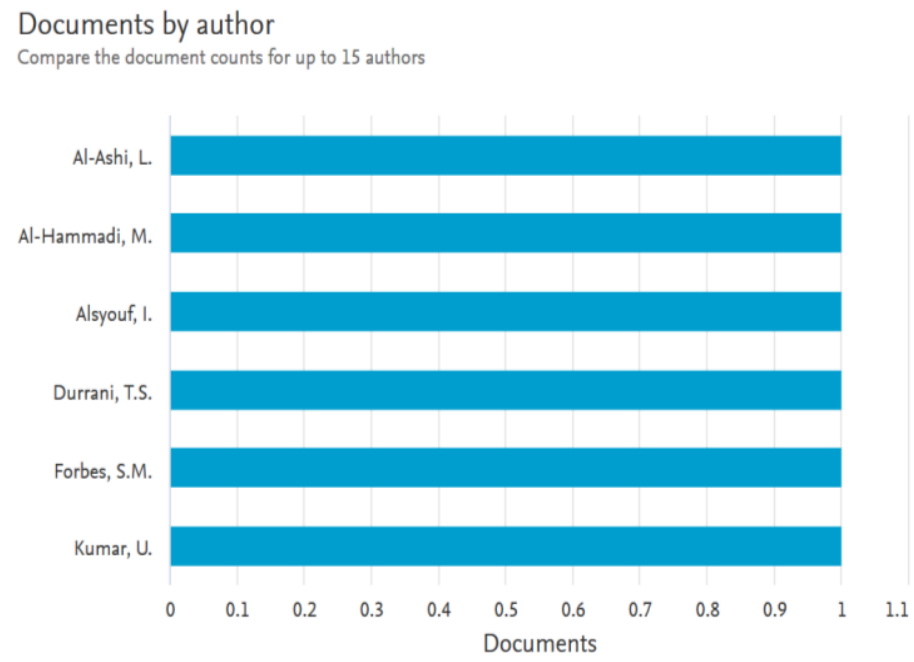

Fuente: Elaboración propia con datos de Scopus (2018).

\section{Categoría 4: Documentos generados por filiación institucional}

En cuanto a las filiaciones instituciones, en la figura 13 se aprecia que la Hong Kong Polytechnic University cuenta con 138 documentos, de los cuales el más referenciado tiene 318 citas (titulado Selección global de proveedores: un enfoque difuso de AHP). En cambio, en la figura 14 se observa que instituciones como Lulea Tekniska Universitet, University of Strathclyde y University of Sharjah tienen un solo documento. 


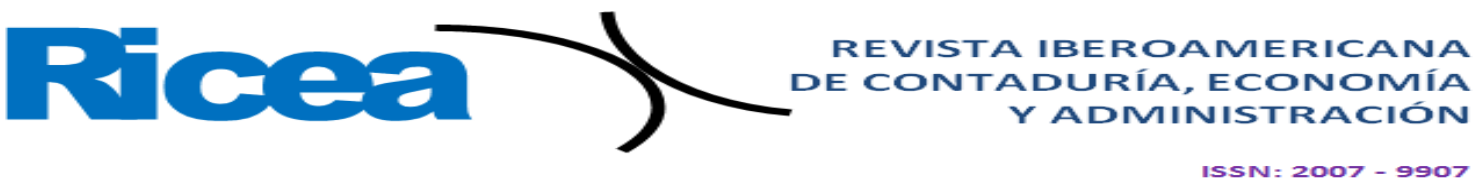

Figura 13. Filiaciones institucionales con más documentos publicados para el criterio Critical success factors

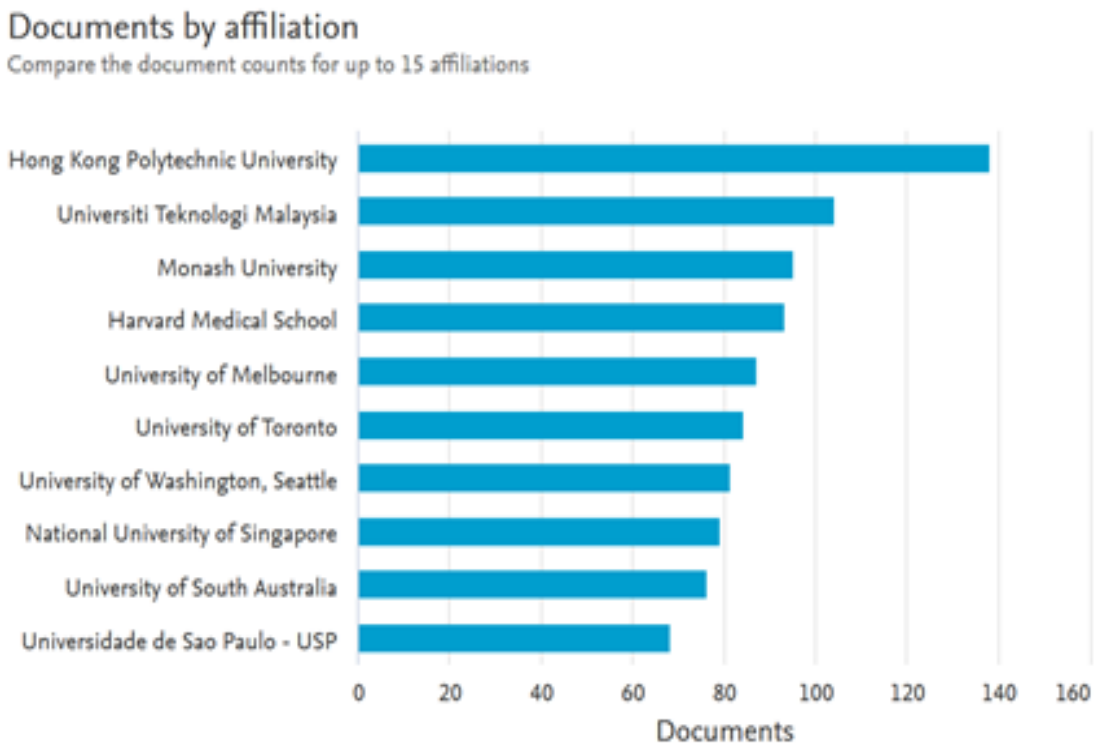

Fuente: Elaboración propia con datos de Scopus (2018).

Figura 14. Filiaciones institucionales con menos documentos publicados para el criterio Critical success factors

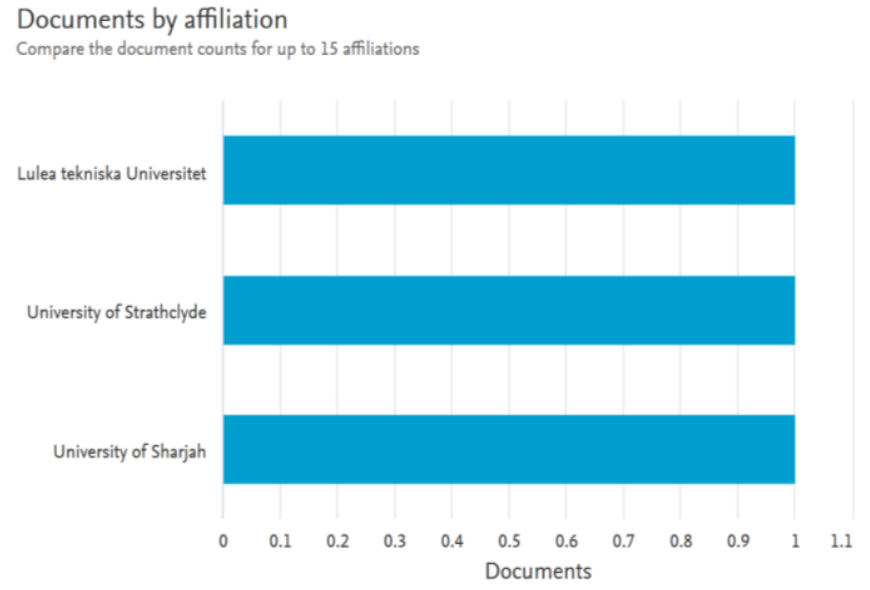

Fuente: Elaboración propia con datos de Scopus (2018).

\section{Categoría 5: Documentos generados por país}

Estados Unidos es el país que cuenta con más documentos (4957) sobre el tema de factores críticos de éxito, mientras que sobre el tema de nuestro interés solo se encontró un texto en Reino Unido y otro que aún no se determina a cuál país pertenece. 


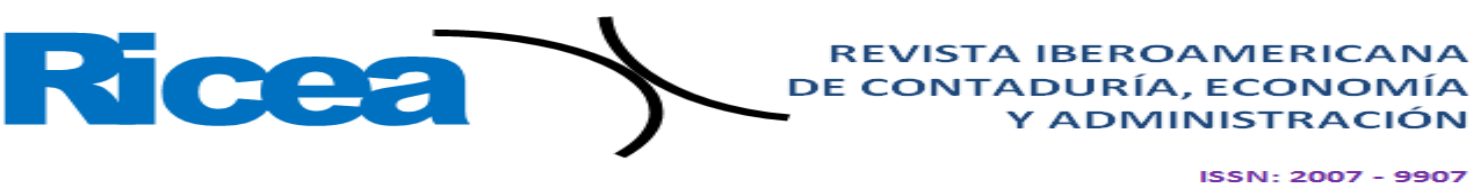

Figura 15. Países con mayor número de documentos generados bajo el criterio Critical success factors

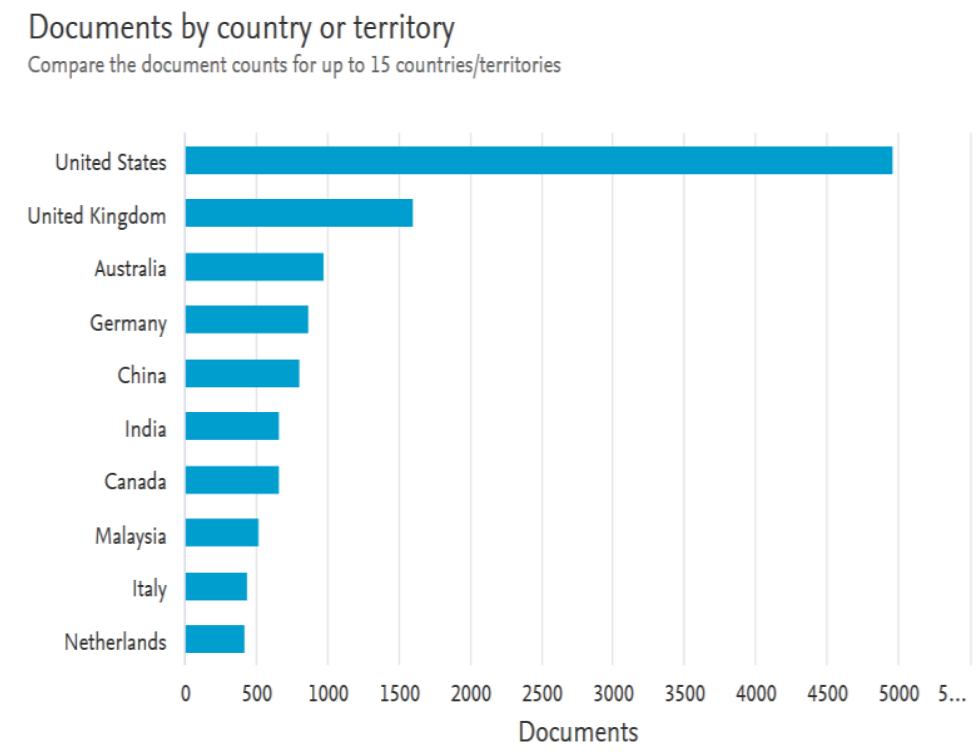

Fuente: Elaboración propia con datos de Scopus (2018).

Figura 16. Países con menor número de documentos generados para el criterio Critical success factors

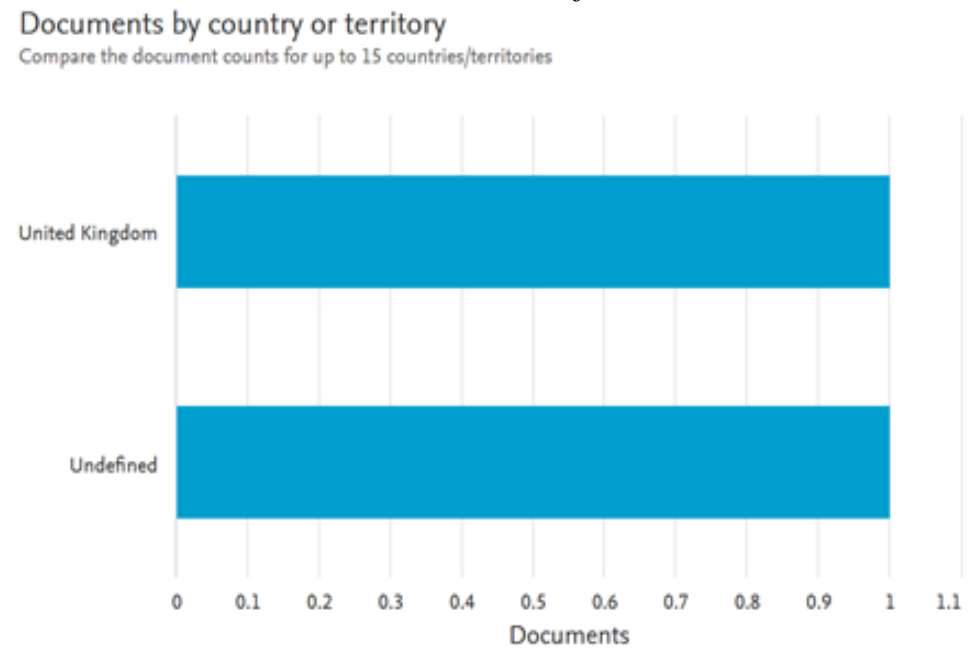

Fuente: Elaboración propia con datos de Scopus (2018).

\section{Categoría 6: Documentos generados por tipo de documento}

Indiscutiblemente, predomina la producción científica en formato de artículos de investigación (57.5\%) y memorias en congresos (24.2\%), lo cual se observa tanto en la figura 17 como en la 18. En esta última, la fuente de la memoria en congreso fue IEEE 


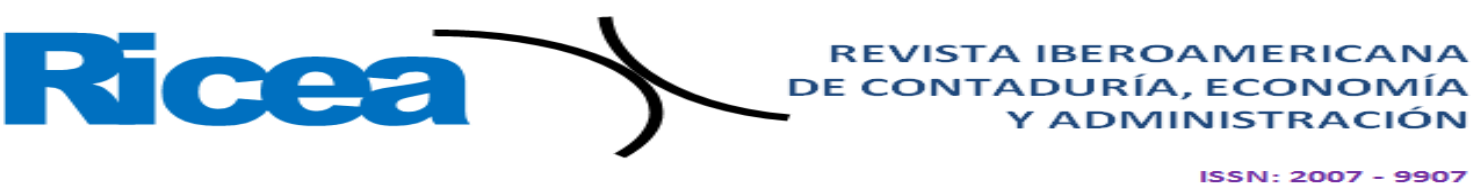

International Engineering Management Conference y la del artículo en revista fue Quality Engineering.

Figura 17. Documentos generados por tipo de documento para el criterio Critical success factors

Documents by type

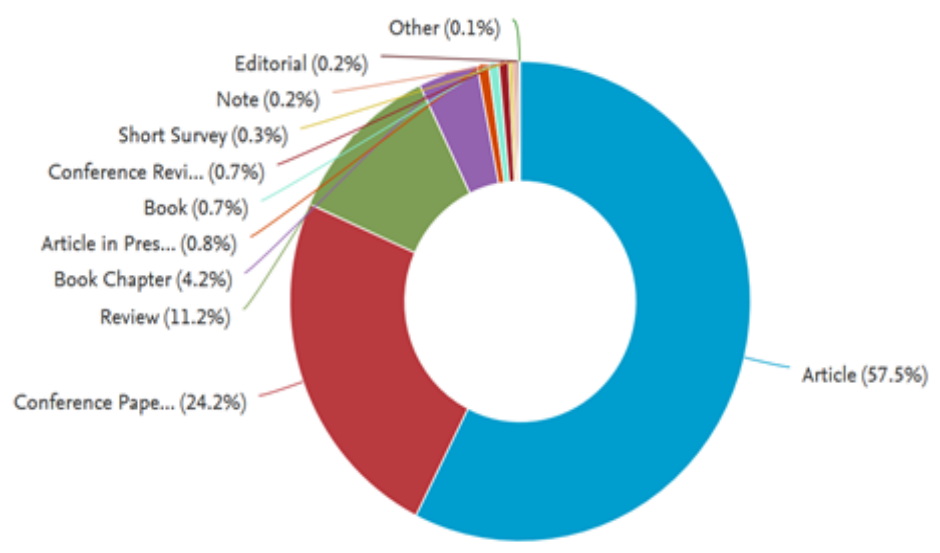

Fuente: Elaboración propia con datos de Scopus (2018).

Figura 18. Documentos generados por tipo de documento (otras fuentes) para el criterio Critical success factors Documents by type

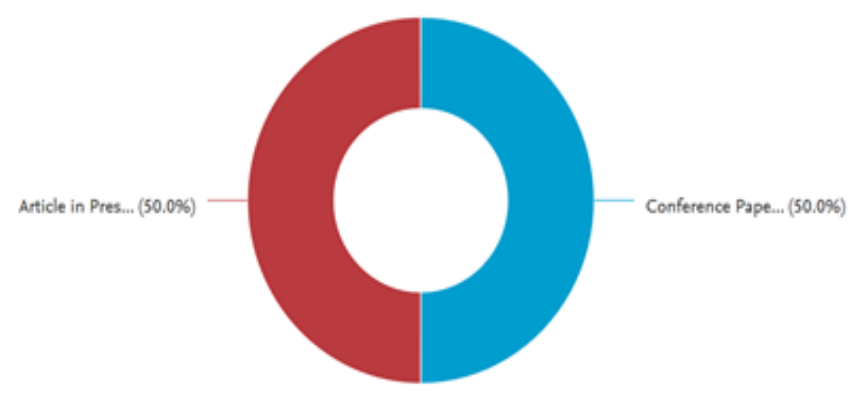

Fuente: Elaboración propia con datos de Scopus (2018) Nota: la fuente de la memoria en congreso fue IEEE International Engineering Management

Conference y la del artículo en revista fue Quality Engineering 


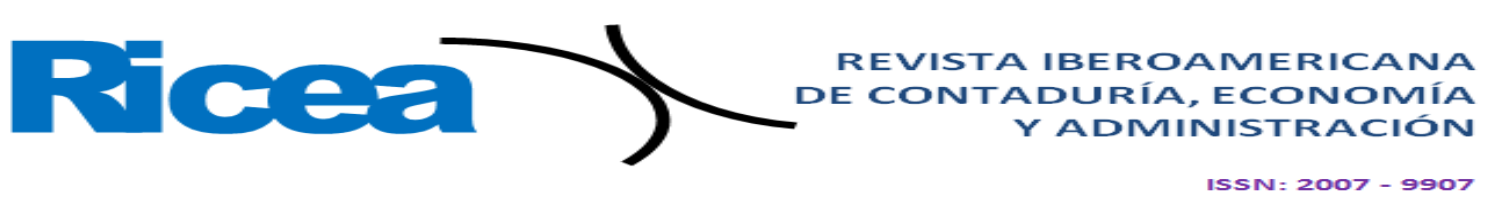

\section{Categoría 7: Documentos generados por área del conocimiento}

La revisión bibliométrica realizada concluye con las figuras 19 y 20, donde se aprecia que, de manera general, se está escribiendo en mayores porcentajes sobre temas de factores críticos de éxito en la gestión de negocios (con 13.6\%). Asimismo, de las 10 áreas de conocimiento en las cuales se pueden ubicar el total de documentos hallados, se puede indicar que en el área de gestión de negocios y contabilidad (Busines, Management and Accounting) está la mayor cantidad de obras (3560 documentos) y el área de medicina (con 3488 documentos).

Por otra parte, en la figura 20 solo se observan dos áreas de conocimiento: ingeniería (66.7\% de los documentos - es decir, 2 - cuyos temas centrales son las aerolíneas) y toma de decisiones (un documento - que representa 33.3\%-, el cual también se enfoca en el tema de las aerolíneas). Esto demuestra que son escasos los artículos especializados sobre el tema de interés en la base de datos consultada (Scopus), pues la mayoría se halla en otras bases menos prestigiosas, lo que genera un vacío cualitativo en cuanto al tema de los factores críticos de éxito en las aerolíneas de bajo costo.

Figura 19. Documentos generados por área del conocimiento para el criterio Critical success factors

Documents by subject area

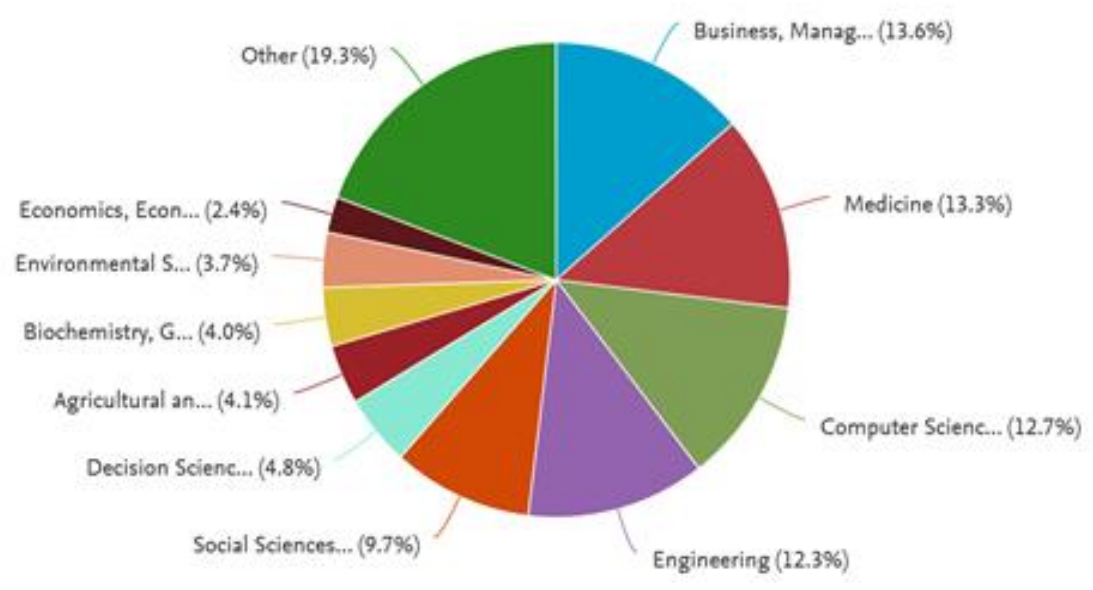

Fuente: Elaboración propia con datos de Scopus (2018). 


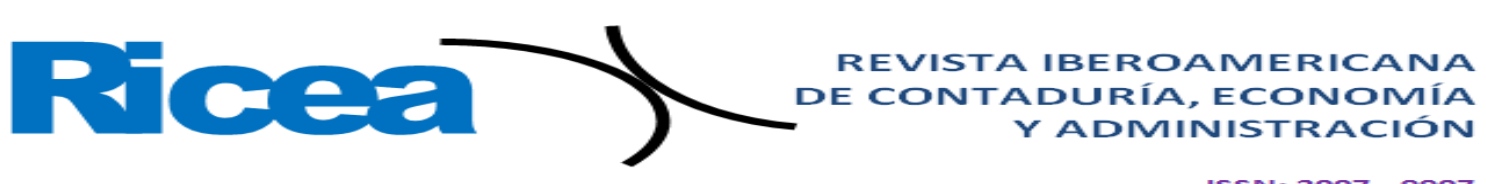

Figura 20. Documentos generados en áreas de ingeniería y toma de decisiones para el criterio Critical success factors

Documents by subject area

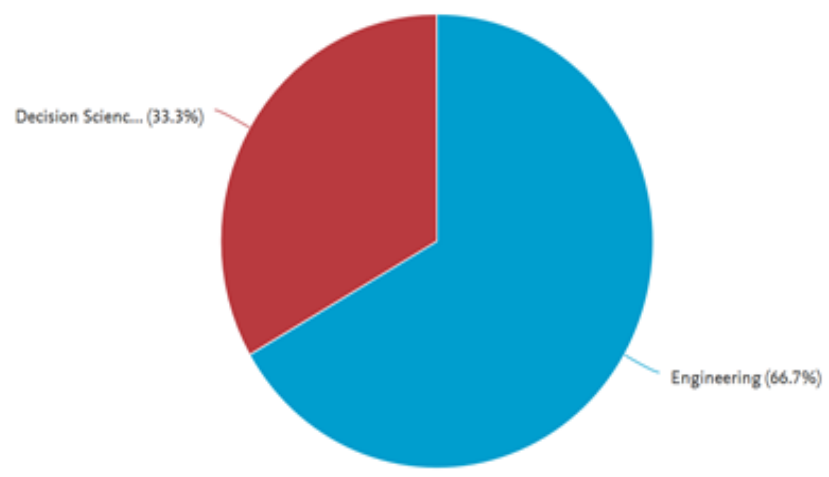

Fuente: Elaboración propia con datos de Scopus (2018).

Como se indicó antes en la figura 19, en el área de negocios se encuentra un total de 3560 documentos, los cuales están distribuidos de esta manera: 50 \% en gestión de negocios y el resto en áreas vinculadas con las ciencias sociales y las humanidades (figura 21).

Figura 21. Documentos generados, en ciencias sociales y otras áreas, para el criterio Critical success factors

Documents by subject area

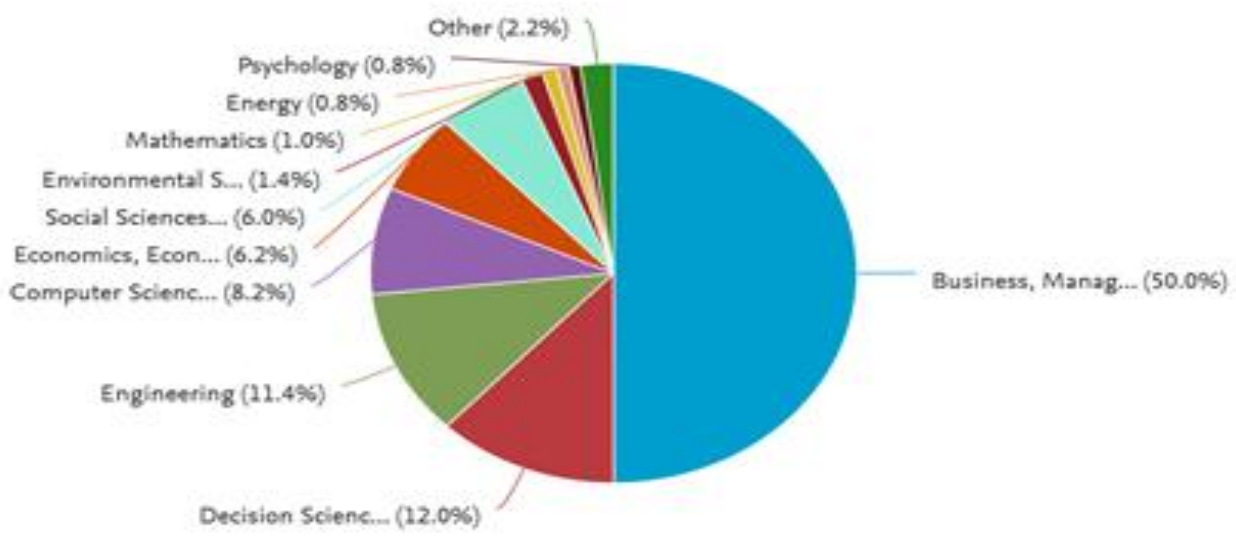

Fuente: Elaboración propia con datos de Scopus (2018).

Con los resultados recabados se comprobó que sí existe una relación muy trascendental entre el liderazgo en costo y el éxito de las aerolíneas de bajo costo. 


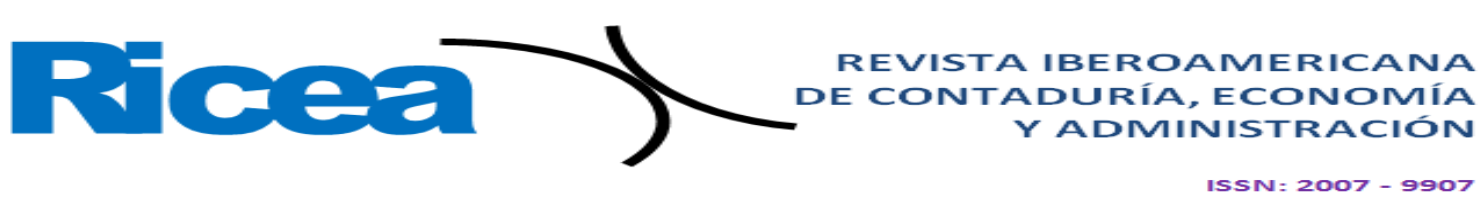

\section{Conclusiones}

Las aerolíneas de bajo costo han beneficiado a los pasajeros, ya que hicieron que el transporte aéreo se convirtiera en una posibilidad para la mayoría de las personas, aunque sacrificando algunas comodidades vinculadas con la alimentación, la elección del asiento, entre otros. Aun así, las compañías de bajo costo en Europa están teniendo mucho éxito basadas en su competitividad y liderazgo, herramientas que les han servido para alcanzar el desarrollo económico.

Por otra parte, y de acuerdo con lo analizado en la presente investigación, se puede concluir que la competitividad es un elemento imprescindible para las grandes y pequeñas organizaciones aéreas que buscan mantener y mejorar una determinada posición en el entorno socioeconómico en el que actúan. En tal sentido, vale destacar que la estandarización aplicada con el uso de un único modelo de avión les está dando una ventaja.

Igualmente, las organizaciones deben identificar los factores críticos de éxito, pues de ese modo podrán gestionar el cumplimiento de sus objetivos planeados, lo cual también brinda la oportunidad de continuar con otras investigaciones mediante la implementación de un modelo de simulación de la red aeroportuaria en Europa o de otros países donde también operan las aerolíneas de bajo costo. Esto contribuirá a que en el futuro sigan apareciendo nuevas áreas de oportunidad para aumentar la competitividad de las compañías de bajo costo.

Otro aspecto pendiente por examinar es el comportamiento de los destinos con mayor número de operaciones de este tipo de empresas en Europa, Estados Unidos de América, México o cualquier otra nación, así como la búsqueda de potenciales rutas para incrementar el número de vuelos. 


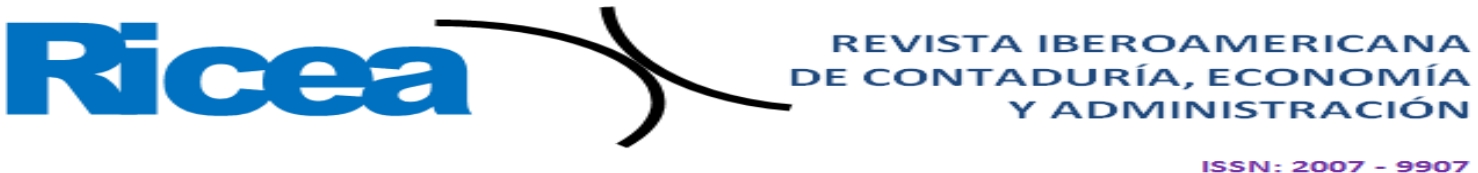

\section{Referencias}

Airbus (2014). Global market forecast 2014-2033. Retrieved from http://www.airbus.com.

Anderson, D., Sweeney, D. y Williams, T. (1999). Estadística para administración y economía. México: International Thomson Editores.

Banco Mundial [BM] (2013). Transporte aéreo. Recuperado de http://datos.bancomundial.org/indicador/IS.AIR.PSGR.

Barbot, C. (2004). Low cost carriers, secondary airports and State aid: an ecnomic assement of the Charleroi affair. FEP Working Papers, (159), 1-20. Retrieved from http://wps.fep.up.pt/wps/wp159.pdf.

Belobaba, P., Odoni, A. and Barnhart, C. (2009). The Global Airline Industry. United Kingdom: Wiley.

Binggeli, U. and Pompeo, L. (2002). Hyped hopes for Europe's low-cost airlines. The McKinsey Quarterly, (4).

Cáceres, J. J. (2006). Conceptos básicos de estadística para ciencias sociales. Las Rozas, Madrid: Delta Publicaciones.

Calder, S. (2002). No frills. The truth behind the low cost revolution in the skies. Virgin Books. London.

Camps, M. and Chauhan, P. (eds.). (2009). Sourcebook of paleolithic transitions: Methods, theories and interpretations. Nueva York: Springer.

Candela-Garriga, J. (2008). El bajo coste y la nueva aviación comercial. Ingeniería y Territorio, (83), 80-89. Recuperado de http://dialnet.unirioja.es/servlet/articulo?codigo=2712712.

Canseco, A., Zúñiga, C. and Blanco, L. (2015). Strategic analysis of Mexico's low-cost airlines development. Revista Electrónica Nova Scientia, 7(15), 343-363.

Cruz, I. (2011). Aerolíneas de bajo costo en México Competencia modal, intermodal e intramodal. Contaduría y Administración, 57(4), 235-251

De la Fuente, S. y Muñoz, C. (2003). Ventaja competitiva: ¿actividades o recursos? Panorama Socioeconómico, (26), Recuperado de http://www.panorama.utalca.cl/dentro/2003-may/estrategiaempresas\%5B1\%5D.pdf

De Rus, G. y Nombela, J. (2003). Economía del transporte. Barcelona: Antoni Bosch. 


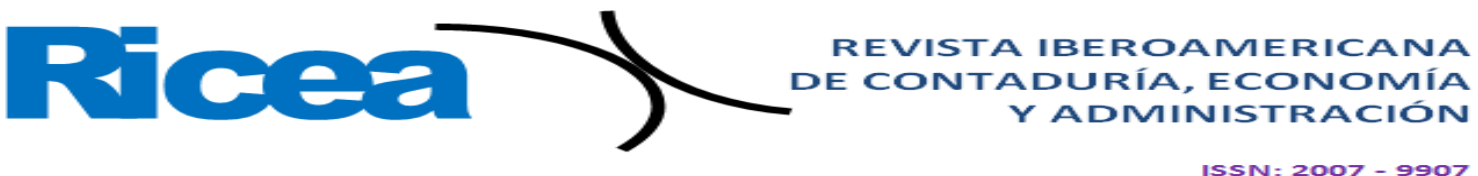

Delgado López-Cózar, E., Ruiz, R. y Jiménez, E. (2006). La edición de revistas científicas: directrices, criterios y modelos de evaluación. Granada: Fundación Española para la Ciencia y la Tecnología. Recuperado de http://recyt. fecyt.es/documentos/Fecyt.pdf. Eurocontrol (2014). Datos y estadísticas históricas. European Organisation for the Safety of Air Navigation.

European Cockpit Association [ECA] (2006). Upheaval in the European Skies. Low cost carriers in Europe: Economic Data, Market and Pilot Demand Forecast. European Cockpit Association. Brussels.

European Low Fares Airline Association [ELFAA] (2004). Liberalisation of European air transport: The benefits of low fare airlines to consumers, airports, regions and the environment. European Low Fares Airline Association.

Francesc, J. (2008). Factores de éxito de las compañías de bajo precio. Estudios Turísticos, (175), 59-79.

Hernández, G. (2000). Competitividad y éxito. Algunas tendencias actuales y su repercusión para el sector empresarial. CEDE.

Hernández, R., Fernández, C. y Baptista, P. (1998). Metodología de la investigación. México: McGraw-Hill.

International Civil Aviation Organization [ICAO] (2014). International Civil Aviation Organization.

Ito, H. and Lee, D. (2003). Low cost carrier growth in the U.S. airline industry: past, present, and future. Working Paper, Brown University, Department of Economics.

Kim, E., Nam, D. and Stimpert, J. (2004). Testing the applicability of porter's generic strategies in the digital age: a study of korean cyber malls. Journal of Business Strategies, 21, 19-45.

Kua, J. and Baum, T. (2004). Perspectives on the development of low cost airlines in Southeast Asia: Evidence from the regional press. Current Issues in Tourism, 7, 262276.

Lizzi, L. (2004). Estrategias competitivas aéreas. Anuario de Estudios en Turismo, 4. Argentina.

McCartney, S. (15 de junio de 2012). ¿Cómo gastan las aerolíneas lo que usted paga por un pasaje? Wall Street Journal. Recuperado de https://www.wsj.com/articles/SB10001424052702303822204577468931588846146 


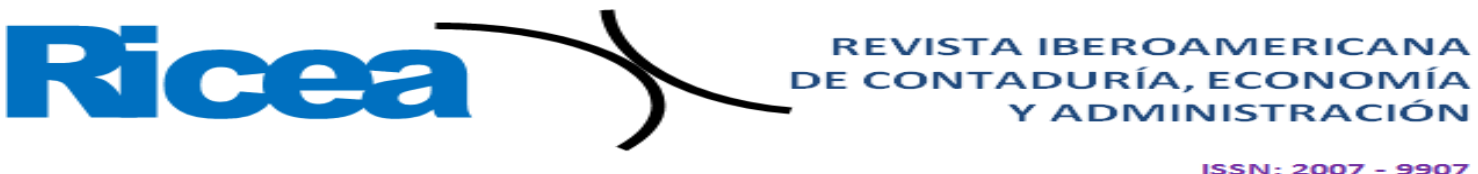

Pender, L. and Baum, T. (2000). Have the frills really left the airline industry? International Journal of Tourism Research, 2(6), 423-436.

Porter, M. (1980). Competitive strategy; techniques for analyzing industries and competitors. New York, Free Press.

Porter, M. (1990). The competitive advantage of nations. Harvard Business Review, 68(2), pp. 73-91.

Porter, M. (1991). La ventaja competitiva de las naciones. Barcelona: Plaza y Janés Editores.

Porter, M. (2000). Estrategias competitivas. México CECSA.

Porter, M. (2007). La ventaja competitiva de las naciones. Harvard Business Review, 85(11), 69-95.

Quero, L. (2008). Estrategias competitivas: factor clave de desarrollo. Negotium: Revista de Ciencias Gerenciales, (10), 36-49.

Quirós Tomás, F. J. (2007). Low cost carriers and tourism: the Spanish case en Using events and festivals to develop tourism. V research meeting among Spanish and Czech academics, Sevilla.

Quirós Tomás, F. J. (2008). European low cost carriers and typical carriers: a comparison. Entrepreneurship and small business in international aspect. Vojtech Korab. Brno.

Quirós Tomás, F. J. (2009). Análisis de la estrategia competitiva de las líneas aéreas europeas. II Jornadas de Investigación en Turismo, Sevilla.

Quirós Tomás, F. J. y Vega Vázquez, M. (2011). Estrategias de fijación de precios en el transporte aéreo de pasajeros. IV Jornadas de Investigación en Turismo, Sevilla.

Sancho, R. (1990). Indicadores bibliométricos utilizados en la evaluación de la ciencia y la tecnología. Revisión bibliográfica. Revista Española de Documentación Científica, $13(3-4), 842-865$.

Scopus (2018). Recuperada de https://www.scopus.com/search/form.uri?display=basic

Secretaría de Comunicación y Transporte [SCT] (2013). Anuario estadístico. Dirección General de Aeronáutica Civil.

Secretaría de Estado de Transportes (2008). Análisis comparativo de costes de escala en los principales aeropuertos europeos. Informe del Área de Estudios y Normas. Dirección General de Aviación Civil. Octubre, España: Ministerio de Fomento.

Skeels, J. (2005a). Challenges facing low fairs airlines the european perspective. Singapur: Elfaa. 
Skeels, J. (2005b). The role of no-frills carriers in the devolopment of Europe's air transport market. Edimburg: Elfaa.

Skeels, J. (2005c). Low fares airlines-competing or complementing? Londres: Elfaa.

Subdirección General de Conocimiento y Estudios Turísticos (2013). Encuesta de gasto turístico 2012. España: Instituto de Estudios Turísticos. Centro de Publicaciones del Ministerio de Industria, Energía y Turismo.

Wernerfelt, B. (1984). A resource based view of the firm. Strategic Management Journal, 5(2), 171-180.

Wernerfelt, B. (1995). The resource based view of the firm: Ten years after. Strategic Management Journal, 16(3), 171-174.

Winston, C. (1998). U. S: industry adjustment to economic deregulation. Journal of Economic Perspectives, 12(3), 89-110. Retrieved from www.airportspotting.com/goodbye-easyjet-737s/. 


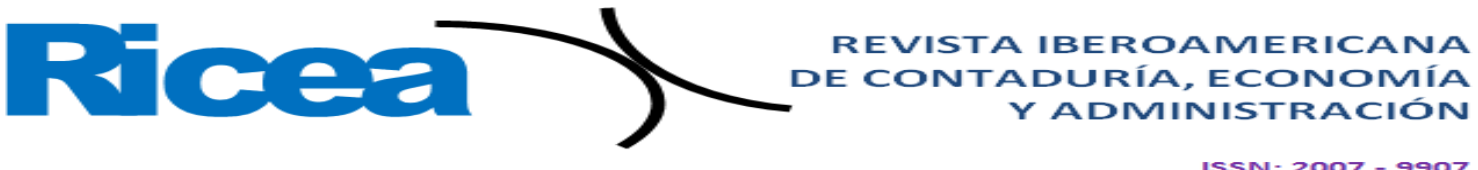

ISSN= $2007-9907$

\begin{tabular}{|c|c|}
\hline Rol de Contribución & Autor (es) \\
\hline Conceptualización & $\begin{array}{l}\text { Carlos Vázquez Cid de León (Principal-igual) Ivonne Maya } \\
\text { Espinoza (Principal-igual) Eric Amín Ramírez Castillo (apoya) }\end{array}$ \\
\hline Metodología & $\begin{array}{l}\text { Carlos Vázquez Cid de León (Principal-igual) Ivonne Maya } \\
\text { Espinoza (Principal-igual) Eric Amín Ramírez Castillo (apoya) }\end{array}$ \\
\hline Software & Carlos Vázquez Cid de León (Principal) \\
\hline Validación & $\begin{array}{l}\text { Carlos Vázquez Cid de León (Principal-igual) Ivonne Maya } \\
\text { Espinoza (Principal-igual) Eric Amín Ramírez Castillo (apoya) }\end{array}$ \\
\hline Análisis Formal & $\begin{array}{l}\text { Carlos Vázquez Cid de León (Principal-igual) Ivonne Maya } \\
\text { Espinoza (Principal-igual) Eric Amín Ramírez Castillo (apoya) }\end{array}$ \\
\hline Investigación & $\begin{array}{l}\text { Carlos Vázquez Cid de León (Principal-igual) Ivonne Maya } \\
\text { Espinoza (Principal-igual) }\end{array}$ \\
\hline Recursos & $\begin{array}{l}\text { Carlos Vázquez Cid de León (Principal-igual) Ivonne Maya } \\
\text { Espinoza (Principal-igual) Eric Amín Ramírez Castillo (apoya) }\end{array}$ \\
\hline Curación de datos & $\begin{array}{l}\text { Carlos Vázquez Cid de León (Principal-igual) Ivonne Maya } \\
\text { Espinoza (Principal-igual) Eric Amín Ramírez Castillo (apoya) }\end{array}$ \\
\hline $\begin{array}{l}\text { Escritura - Preparación } \\
\text { del borrador original }\end{array}$ & $\begin{array}{l}\text { Carlos Vázquez Cid de León (Principal-igual) Ivonne Maya } \\
\text { Espinoza (Principal-igual) Eric Amín Ramírez Castillo (apoya) }\end{array}$ \\
\hline $\begin{array}{l}\text { Escritura - Revisión y } \\
\text { edición }\end{array}$ & $\begin{array}{l}\text { Carlos Vázquez Cid de León (Principal-igual) Ivonne Maya } \\
\text { Espinoza (Principal-igual) Eric Amín Ramírez Castillo (apoya) }\end{array}$ \\
\hline Visualización & $\begin{array}{l}\text { Carlos Vázquez Cid de León (Principal-igual) Ivonne Maya } \\
\text { Espinoza (Principal-igual) Eric Amín Ramírez Castillo (apoya) }\end{array}$ \\
\hline Supervisión & $\begin{array}{l}\text { Carlos Vázquez Cid de León (Principal-igual) Ivonne Maya } \\
\text { Espinoza (Principal-igual) }\end{array}$ \\
\hline $\begin{array}{l}\text { Administración de } \\
\text { Proyectos }\end{array}$ & $\begin{array}{l}\text { Carlos Vázquez Cid de León (Principal-igual) Ivonne Maya } \\
\text { Espinoza (Principal-igual) }\end{array}$ \\
\hline Adquisición de fondos & Carlos Vázquez Cid de León (Principal) \\
\hline
\end{tabular}

